\title{
C-H Bond Activation of Hydrocarbons by an Imidozirconocene Complex
}

\author{
Helen M. Hoyt, Forrest E. Michael, and Robert G. Bergman* \\ Department of Chemistry, University of California, Berkeley, Berkeley, CA 94720
}

\section{Supporting Information}

General Procedures. All air- and moisture-sensitive compounds were manipulated using standard Schlenk techniques, or in a glovebox under an inert atmosphere $\left(\mathrm{N}_{2}\right)$. Glassware was dried overnight at $150^{\circ} \mathrm{C}$ or flame dried under vacuum immediately prior to use. Sealed NMR tubes were prepared by attaching the NMR tube directly to a Kontes high-vacuum stopcock via a Cajon Ultra-Torr reducing union and then flame-sealing on a vacuum line. All NMR spectra were obtained at ambient temperature using Bruker AMX-400, AVB-400, AVQ400 , or DRX-500 spectrometers. ${ }^{1} \mathrm{H}$ NMR chemical shifts $(\delta)$ are reported in parts per million (ppm) downfield of TMS and are referenced relative to residual protiated solvent. ${ }^{13} \mathrm{C}\left\{{ }^{1} \mathrm{H}\right\}$ NMR chemical shifts $(\delta)$ are reported in ppm relative to the carbon resonance of the deuterated solvent. In cases where assignment of ${ }^{13} \mathrm{C}$ resonances was ambiguous, assignments were made by analysis of spectra from standard DEPT 135 pulse sequences and, in some cases, additional 2D NMR experiments (NOESY, ${ }^{1} \mathrm{H}-{ }^{13} \mathrm{C}$ HMQC, etc). ${ }^{19} \mathrm{~F}$ NMR spectra were recorded at 377 $\mathrm{MHz}$, and chemical shifts $(\delta)$ are reported relative to external $\mathrm{CFCl}_{3}$. Elemental analyses were performed at the University of California, Berkeley Microanalytical facility. X-ray structures were obtained at the UC Berkeley CHEXRAY facility and the structures were solved by Dr. Fred Hollander, Dr. Allen Oliver, and Dennis Leung. Note: purification and full characterization of some of these complexes has been difficult due to their extreme solubility in pentane and other solvents, particularly those complexes with alkyl ligands. Further investigation into purification of these complexes is currently underway. Additionally, we have yet to have success in analyzing these complexes by mass spectrometry.

Materials. Unless otherwise noted, reagents were purchased from commercial suppliers and used without further purification. Silica gel (Merck 60, 230-400) was dried under vacuum at $250{ }^{\circ} \mathrm{C}$ for $48 \mathrm{~h}$. Pentane, hexane, benzene, and toluene (Fisher) were passed through a column of activated Alumina (type A2, size $12 \times 32$, Purify Co.) under nitrogen pressure and sparged with $\mathrm{N}_{2}$ prior to use. ${ }^{1}$ Unless otherwise noted, all other hydrocarbons and deuterated solvents (Cambridge Isotope Laboratories) were either dried over calcium hydride and vacuum transferred to a glass vessel equipped with a vacuum stopcock and stored over $3 \mathrm{~A}$ molecular sieves, or were degassed by three freeze-pump-thaw cycles and were dried over $3 \mathrm{~A}$ molecular sieves. Hydrocarbon reagents were additionally passed through a plug of activated silica gel prior to use. Diethyl ether and tetrahydrofuran (Fisher) were distilled from purple sodium/benzophenone ketyl under $\mathrm{N}_{2}$ prior to use.

General Experimental Details for X-ray Structure Determinations. A fragment of a crystal was mounted onto a glass fiber using Paratone $\mathrm{N}$ hydrocarbon oil and was transferred to a Siemens SMART ${ }^{2}$ CCD area detector with graphite monochromated Mo-K $\alpha$ radiation. The crystal was centered in the beam and cooled by a nitrogen-flow low-temperature apparatus to $-150{ }^{\circ} \mathrm{C}$. Cell constants and orientation matrices were obtained from a least-squares refinement 
using measured positions (1c: 2527 reflections in the range $5.32<2 \theta<46.85^{\circ}$; 1d: 1902 reflections in the range $4.70<2 \theta<42.90^{\circ} ; \mathbf{3}: 6716$ reflections in the range $3.50<2 \theta<45.00^{\circ}, \mathbf{4}$ : 4458 reflections in the range $4.70<2 \theta<42.00^{\circ}$ ). Frames corresponding to an arbitrary hemisphere of data were collected using $\omega$ scans of $0.3^{\circ}$ counted for a total of 10.0 seconds per frame. The raw data were integrated by the program $\mathrm{SAINT}^{3}$ to a maximum $2 \theta$ value of 1c: $49.6^{\circ}, \mathbf{1 d}: 49.4^{\circ}, \mathbf{3}: 49.3^{\circ}$, and $4: 49.5^{\circ}$. The data were corrected for Lorentz and polarization effects and were analyzed for agreement and possible absorption using XPREP. ${ }^{4}$ An empirical absorption correction was applied using $\operatorname{SADABS}^{5}(\operatorname{Tmax}=1.00, \mathbf{1 c}: \mathrm{Tmin}=0.60 ; \mathbf{1 d}$ : $\mathrm{Tmin}=$ $0.51 ; 4: \operatorname{Tmin}=0.55,3: \mathrm{Tmax}=0.98, \mathrm{Tmin}=0.63$ ). The structure was resolved by direct methods and expanded using Fourier techniques. The quantity minimized by the least squares program was $\Sigma_{w}\left(|| \mathrm{F}_{o}|-| \mathrm{F}_{c} \mid\right)^{2}$, where $w$ is the weight of a given observation. The weighting scheme was based on counting statistics and included a factor $(\mathrm{p}=0.030)$ to downweight the intense reflections. The analytical forms of the scattering factor tables for the neutral atoms were used, and all scattering factors were corrected for both the real and imaginary components of anomalous dispersion. All calculations were performed using the teXsan crystallographic software package of the Molecular Structure Corporation. ${ }^{6}$

rac-(ebthi) $\mathbf{Z r}\left(\mathbf{N H C M e}_{3}\right)\left(\mathbf{C H}_{3}\right)$ (1a). The $t$-butyl amide complex 1a was synthesized in the same manner as the known aryl amide complex by adding lithium $t$-butyl amide to the methyl chloride complex. ${ }^{7} \quad$ A $1000-\mathrm{mL}$ roundbottom Schlenk flask was charged with rac(ebthi)Zr(Me)(Cl) (3.34 g, $8.24 \mathrm{mmol})$, THF (200 mL), and lithium $t$-butyl amide $(0.652 \mathrm{~g}, 8.24$ mmol) in the glovebox. The flask was attached to a Schlenk line and the reaction mixture was stirred for $1.5 \mathrm{~h}$ under positive $\mathrm{N}_{2}$ pressure at ambient temperature. THF was then removed under reduced pressure. Pentane $(400 \mathrm{~mL})$ was added to the flask via cannula, and the mixture was stirred for $2 \mathrm{~h}$ under positive $\mathrm{N}_{2}$ pressure at ambient temperature. The pentane solution was transferred to a 500-mL Schlenk flask with a cannula filter, leaving the insoluble lithium chloride behind. The pentane solution was concentrated to $150 \mathrm{~mL}$ under vacuum and the flask was then placed in a $-80{ }^{\circ} \mathrm{C}$ freezer for $6 \mathrm{~h}$ for crystallization. The pentane solution was removed from the resulting yellow product by cannula filtration at $-78{ }^{\circ} \mathrm{C}$, and excess solvent was removed from the product under reduced pressure to yield complex $\mathbf{1 a}$ as an analytically pure light yellow powder $(3.06 \mathrm{~g}, 84 \%) .{ }^{1} \mathrm{H}$ NMR $(500 \mathrm{MHz}) \delta 6.12(\mathrm{~d}, J=2.9 \mathrm{~Hz}, 1 \mathrm{H}, \mathrm{CpH}), 5.86(\mathrm{~d}, J=2.9 \mathrm{~Hz}$, $1 \mathrm{H}, \mathrm{CpH}), 5.44(\mathrm{~d}, J=3.1 \mathrm{~Hz}, 1 \mathrm{H}, \mathrm{CpH}), 5.06(\mathrm{~d}, J=3.1 \mathrm{~Hz}, 1 \mathrm{H}, \mathrm{CpH}), 4.66(\mathrm{~s}, 1 \mathrm{H}, \mathrm{NH}), 2.8-$ $2.6(\mathrm{~m}, 2 \mathrm{H}), 2.6-2.3(\mathrm{~m}, 8 \mathrm{H}), 2.2-2.1(\mathrm{~m}, 1 \mathrm{H}), 1.9-1.6(\mathrm{~m}, 4 \mathrm{H}), 1.6-1.5(\mathrm{~m}, 1 \mathrm{H}), 1.5-1.4(\mathrm{~m}, 2 \mathrm{H})$, 1.3-1.2 (m, 2H), $1.18(\mathrm{~s}, 9 \mathrm{H}, \mathrm{CMe}), 0.03\left(\mathrm{~s}, \mathrm{ZrCH}_{3}\right) \mathrm{ppm} .{ }^{13} \mathrm{C}\left\{{ }^{1} \mathrm{H}\right\} \mathrm{NMR}(125 \mathrm{MHz}) \delta 126.90$ $\left(\mathrm{ebthi}_{\mathrm{q}}\right), 125.09\left(\mathrm{ebthi}_{\mathrm{q}}\right), 123.72\left(\mathrm{ebthi}_{\mathrm{q}}\right), 123.56\left(\mathrm{ebthi}_{\mathrm{q}}\right), 119.87\left(\mathrm{ebthi}_{\mathrm{q}}\right), 117.43\left(\mathrm{ebthi}_{\mathrm{q}}\right), 110.97$ $\left.(\text { ebthi })_{\mathrm{t}}\right), 105.96\left(\mathrm{ebthi}_{\mathrm{t}}\right), 105.54\left(\mathrm{ebthi}_{\mathrm{t}}\right), 101.20\left(\mathrm{ebthi}_{\mathrm{t}}\right), 55.55\left(\mathrm{HNCMe}_{3}\right), 34.84\left(\mathrm{HNCMe}_{3}\right)$, $27.98\left(\mathrm{CH}_{2}\right), 27.45\left(\mathrm{CH}_{2}\right), 24.87\left(\mathrm{CH}_{2}\right), 24.54\left(\mathrm{CH}_{2}\right), 23.79\left(\mathrm{CH}_{2}\right), 23.75\left(\mathrm{CH}_{2}\right), 23.63\left(\mathrm{CH}_{2}\right)$, $23.44\left(\mathrm{CH}_{2}\right), 23.22\left(\mathrm{CH}_{2}\right), 23.19\left(\mathrm{CH}_{2}\right), 20.33\left(\mathrm{ZrCH}_{3}\right)$ ppm. Anal. Calcd for $\mathrm{C}_{25} \mathrm{H}_{37} \mathrm{NZr}$ : C, 67.81; H, 8.42; N, 3.16. Found: C, 67.73; H, 8.42; N, 3.0.

rac-(ebthi)Zr(NHCMe $\left.)_{3}\right)(n$-hexyl) (1b). A solution of complex 1a $(0.105 \mathrm{~g}, 0.236$ $\mathrm{mmol})$ in $n$-hexane $(6.0 \mathrm{~mL})$ and was heated in a glass vessel equipped with a vacuum stopcock under an atmosphere of $\mathrm{N}_{2}$ for $24 \mathrm{~h}$ at $75{ }^{\circ} \mathrm{C}$. The volatile materials were removed under vacuum to yield $\mathbf{1 b}$ as an orange oil $\left(0.113 \mathrm{~g}, 93 \%,>95 \%\right.$ pure by ${ }^{1} \mathrm{H}$ NMR spectroscopy (Figure S-1)). Purification of this complex has been hampered by its extreme solubility in pentane and other solvents. ${ }^{1} \mathrm{H}$ NMR $(500 \mathrm{MHz}) \delta 6.12(\mathrm{~d}, J=2.9 \mathrm{~Hz}, 1 \mathrm{H}, \mathrm{CpH}), 6.09(\mathrm{~d}, J=3.1 \mathrm{~Hz}, 1 \mathrm{H}, \mathrm{Cp} H)$, $5.40(\mathrm{~d}, J=3.1 \mathrm{~Hz}, 1 \mathrm{H}, \mathrm{Cp} H), 5.14(\mathrm{~d}, J=3.1 \mathrm{~Hz}, 1 \mathrm{H}, \mathrm{Cp} H), 4.77(\mathrm{~s}, 1 \mathrm{H}, \mathrm{NH}), 2.8-2.7(\mathrm{~m}, 1 \mathrm{H})$, 2.6-2.4 (m, 8H), 2.4-2.3 (m, 4H), 2.2-2.1 (m, 1H), 2.1-1.9 (m, 1H), 1.9-1.8 (m, 2H), 1.8-1.6 (m, 
$3 \mathrm{H}), 1.6-1.4(\mathrm{~m}, 8 \mathrm{H}), 1.21(\mathrm{~s}, 9 \mathrm{H}, \mathrm{CMe}), 1.00\left(\mathrm{t}, J=7.3 \mathrm{~Hz}, 3 \mathrm{H}, \mathrm{CH}_{3}\right), 0.59(\mathrm{td}, J=12.2 \mathrm{~Hz}, J=$ $\left.4.0 \mathrm{~Hz}, 1 \mathrm{H}, \mathrm{ZrCH}_{2} \mathrm{C}_{5} \mathrm{H}_{11}\right), 0.45\left(\mathrm{td}, J=12.1 \mathrm{~Hz}, J=4.6 \mathrm{~Hz}, 1 \mathrm{H}, \mathrm{ZrCH}_{2} \mathrm{C}_{5} \mathrm{H}_{11}\right) \mathrm{ppm} .{ }^{13} \mathrm{C}\left\{{ }^{1} \mathrm{H}\right\}$ NMR (125 MHz) $\delta 126.61\left(\right.$ ebthi $\left._{\mathrm{q}}\right), 124.84\left(\mathrm{ebthi}_{\mathrm{q}}\right), 123.25\left(\mathrm{ebthi}_{\mathrm{q}}\right), 123.10\left(\mathrm{ebthi}_{\mathrm{q}}\right), 119.92$ $\left(\mathrm{ebthi}_{\mathrm{q}}\right), 118.76\left(\mathrm{ebthi}_{\mathrm{q}}\right), 109.67\left(\mathrm{ebthi}_{\mathrm{t}}\right), 105.86\left(\mathrm{ebthi}_{\mathrm{t}}\right), 105.55\left(\mathrm{ebthi}_{\mathrm{t}}\right), 101.26\left(\mathrm{ebthi}_{\mathrm{t}}\right), 55.70$ $\left(\mathrm{HNCMe}_{3}\right), 45.19\left(\mathrm{ZrCH}_{2} \mathrm{C}_{4} \mathrm{H}_{9}\right), 38.18\left(\mathrm{CH}_{2}\right), 36.88\left(\mathrm{CH}_{2}\right), 34.80\left(\mathrm{HNCMe}_{3}\right), 32.53\left(\mathrm{CH}_{2}\right)$, $28.02\left(\mathrm{CH}_{2}\right), 27.14\left(\mathrm{CH}_{2}\right), 24.67\left(\mathrm{CH}_{2}\right), 24.57\left(\mathrm{CH}_{2}\right), 23.79\left(\mathrm{CH}_{2}\right), 23.57\left(\mathrm{CH}_{2}\right), 23.52\left(\mathrm{CH}_{2}\right)$, $23.48\left(\mathrm{CH}_{2}\right), 23.45\left(\mathrm{CH}_{2}\right), 23.26\left(\mathrm{CH}_{2}\right), 23.02\left(\mathrm{CH}_{2}\right), 14.65\left(\mathrm{ZrC}_{4} \mathrm{H}_{8} C_{3}\right)$ ppm.

rac-(ebthi)Zr(NHCMe $)(n$-pentyl) (1c). A solution of complex 1a $(0.101 \mathrm{~g}, 0.228$ mmol) in $n$-pentane $(5.0 \mathrm{~mL})$ was heated in a glass vessel equipped with a vacuum stopcock under an atmosphere of $\mathrm{N}_{2}$ for $24 \mathrm{~h}$ at $75{ }^{\circ} \mathrm{C}$. The volatile materials were removed under vacuum to yield 1c as a yellow oil $\left(0.105 \mathrm{~g}, 92 \%,>95 \%\right.$ pure by ${ }^{1} \mathrm{H}$ NMR spectroscopy (Figure S-2)), which was then crystallized $(80 \%)$ at $-30{ }^{\circ} \mathrm{C}$ from diethyl ether to yield yellow tablets in the acentric, monoclinic space group $\mathrm{P} 2{ }_{1} .{ }^{1} \mathrm{H}$ NMR $(500 \mathrm{MHz}) \delta 6.11(\mathrm{~d}, J=3.1 \mathrm{~Hz}, 1 \mathrm{H}, \mathrm{Cp} H)$, $6.07(\mathrm{~d}, J=2.9 \mathrm{~Hz}, 1 \mathrm{H}, \mathrm{Cp} H), 5.39(\mathrm{~d}, J=3.1 \mathrm{~Hz}, 1 \mathrm{H}, \mathrm{Cp} H), 5.13(\mathrm{~d}, J=2.9 \mathrm{~Hz}, 1 \mathrm{H}, \mathrm{Cp} H)$, $4.76(\mathrm{~s}, 1 \mathrm{H}, \mathrm{NH}), 2.8-2.7(\mathrm{~m}, 1 \mathrm{H}), 2.6-2.4(\mathrm{~m}, 6 \mathrm{H}), 2.4-2.3(\mathrm{~m}, 3 \mathrm{H}), 2.2-2.1(\mathrm{~m}, 1 \mathrm{H}), 2.1-1.9(\mathrm{~m}$, $1 \mathrm{H}), 1.9-1.6(\mathrm{~m}, 6 \mathrm{H}), 1.6-1.4(\mathrm{~m}, 8 \mathrm{H}), 1.20(\mathrm{~s}, 9 \mathrm{H}, \mathrm{CMe} 3), 1.06\left(\mathrm{t}, J=7.0 \mathrm{~Hz}, 3 \mathrm{H}, \mathrm{CH}_{3}\right), 0.58$ $\left(\mathrm{td}, J=11.9 \mathrm{~Hz}, J=4.1 \mathrm{~Hz}, 1 \mathrm{H}, \mathrm{ZrCH}_{2} \mathrm{C}_{4} \mathrm{H}_{9}\right), 0.40$ (td, $\left.J=12.4 \mathrm{~Hz}, J=4.4 \mathrm{~Hz}, 1 \mathrm{H}, \mathrm{ZrCH}_{2} \mathrm{C}_{4} \mathrm{H}_{9}\right)$ ppm. ${ }^{13} \mathrm{C}\left\{{ }^{1} \mathrm{H}\right\}$ NMR (125 MHz) $\delta 126.61$ (ebthi $\left.\mathrm{q}_{\mathrm{q}}\right), 124.84\left(\mathrm{ebthi}_{\mathrm{q}}\right), 123.23\left(\mathrm{ebthi}_{\mathrm{q}}\right), 123.09$ $\left(\mathrm{ebthi}_{\mathrm{q}}\right), 119.90\left(\mathrm{ebthi}_{\mathrm{q}}\right), 118.75\left(\mathrm{ebthi}_{\mathrm{q}}\right), 109.65\left(\mathrm{ebthi}_{\mathrm{t}}\right), 105.86\left(\mathrm{ebthi}_{\mathrm{t}}\right), 105.55\left(\mathrm{ebthi}_{\mathrm{t}}\right), 101.26$ (ebthi $)$, $55.70\left(\mathrm{HNCMe}_{3}\right), 45.03\left(\mathrm{ZrCH}_{2} \mathrm{C}_{4} \mathrm{H}_{9}\right), 40.73\left(\mathrm{CH}_{2}\right), 36.51\left(\mathrm{CH}_{2}\right), 34.80\left(\mathrm{HNCMe}_{3}\right)$, $28.03\left(\mathrm{CH}_{2}\right), 27.14\left(\mathrm{CH}_{2}\right), 24.66\left(\mathrm{CH}_{2}\right), 24.57\left(\mathrm{CH}_{2}\right), 23.80\left(\mathrm{CH}_{2}\right), 23.58\left(\mathrm{CH}_{2}\right), 23.52\left(\mathrm{CH}_{2}\right)$, $23.45\left(\mathrm{CH}_{2}\right), 23.26\left(\mathrm{CH}_{2}\right), 23.21\left(\mathrm{CH}_{2}\right), 23.10\left(\mathrm{CH}_{2}\right), 14.83\left(\mathrm{ZrC}_{4} \mathrm{H}_{8} C_{3}\right) \mathrm{ppm}$. Anal. Calcd for $\mathrm{C}_{29} \mathrm{H}_{45} \mathrm{NZr}$ : C, 69.82; H, 9.09; N, 2.81. Found: C, 69.66; H, 9.06; N, 2.96.

rac-(ebthi)Zr(NHCMe $)\left(\mathrm{CH}_{2} \mathrm{SiMe}_{3}\right)(\mathbf{1 d})$. A solution of complex $1 \mathrm{a}(0.102 \mathrm{~g}, 0.229$ mmol) in tetramethylsilane $(4.0 \mathrm{~mL})$ and cyclohexane $(0.50 \mathrm{~mL})$ was heated in a glass vessel equipped with a vacuum stopcock under an atmosphere of $\mathrm{N}_{2}$ for $24 \mathrm{~h}$ at $75{ }^{\circ} \mathrm{C}$. The volatile materials were removed under vacuum to yield $\mathbf{1 d}$ as a white film $(0.105 \mathrm{~g}, 89 \%,>95 \%$ pure by ${ }^{1} \mathrm{H}$ NMR spectroscopy (Figure S-3)), which was then crystallized at $-30{ }^{\circ} \mathrm{C}$ from toluene to yield colorless crystals. Complex $1 \mathbf{d}$ crystallized in the centric space group $\mathrm{P} 2{ }_{1} / \mathrm{n}$ with one molecule in the asymmetric unit and four molecules in the unit cell. ${ }^{1} \mathrm{H}$ NMR $(500 \mathrm{MHz}) \delta 6.29(\mathrm{~d}, J=2.9$ $\mathrm{Hz}, 1 \mathrm{H}, \mathrm{CpH}), 6.14(\mathrm{~d}, J=2.9 \mathrm{~Hz}, 1 \mathrm{H}, \mathrm{Cp} H), 5.41(\mathrm{~d}, J=3.1 \mathrm{~Hz}, 1 \mathrm{H}, \mathrm{CpH}), 5.13(\mathrm{~d}, J=3.1 \mathrm{~Hz}$, $1 \mathrm{H}, \mathrm{CpH}), 4.81(\mathrm{~s}, 1 \mathrm{H}, \mathrm{NH}), 2.6-2.5(\mathrm{~m}, 2 \mathrm{H}), 2.5-2.4(\mathrm{~m}, 5 \mathrm{H}), 2.4-2.3(\mathrm{~m}, 4 \mathrm{H}), 2.2-2.1(\mathrm{~m}, 1 \mathrm{H})$, 1.8-1.6 (m, 4H), 1.5-1.3 (m, 4H), 1.21 (s, 9H, CMe $), 0.33$ (s, 9H, SiMes), -0.47 (d, J=11.7 Hz, $\left.1 \mathrm{H}, \mathrm{ZrCH}_{2} \mathrm{Si}\right),-0.76\left(\mathrm{~d}, J=11.5 \mathrm{~Hz}, 1 \mathrm{H}, \mathrm{ZrCH}_{2} \mathrm{Si}\right) \mathrm{ppm} .{ }^{13} \mathrm{C}\left\{{ }^{1} \mathrm{H}\right\} \mathrm{NMR}(125 \mathrm{MHz}) \delta 126.69$ $\left(\mathrm{ebthi}_{\mathrm{q}}\right), 126.55\left(\mathrm{ebthi}_{\mathrm{q}}\right), 125.41\left(\mathrm{ebthi}_{\mathrm{q}}\right), 124.23\left(\mathrm{ebthi}_{\mathrm{q}}\right), 120.01\left(\mathrm{ebthi}_{\mathrm{q}}\right), 118.59\left(\mathrm{ebthi}_{\mathrm{q}}\right), 107.53$ $\left.(\text { ebthi })_{\mathrm{t}}\right), 105.82\left(\mathrm{ebthi}_{\mathrm{t}}\right), 104.38\left(\mathrm{ebthi}_{\mathrm{t}}\right), 101.78\left(\mathrm{ebthi}_{\mathrm{t}}\right), 56.09\left(\mathrm{HNCMe}_{3}\right), 34.79\left(\mathrm{HNCMe}_{3}\right)$, $28.08\left(\mathrm{CH}_{2}\right), 27.91\left(\mathrm{CH}_{2}\right), 27.10\left(\mathrm{CH}_{2}\right), 24.71\left(\mathrm{CH}_{2}\right), 24.69\left(\mathrm{CH}_{2}\right), 23.92\left(\mathrm{CH}_{2}\right), 23.67\left(\mathrm{CH}_{2}\right)$, $23.49\left(\mathrm{CH}_{2}\right), 23.41\left(\mathrm{CH}_{2}\right), 23.05\left(\mathrm{CH}_{2}\right), 22.99\left(\mathrm{CH}_{2}\right), 4.41\left(\mathrm{Si}\left(\mathrm{CH}_{3}\right)_{3}\right) \mathrm{ppm}$. Anal. Calcd for $\mathrm{C}_{28} \mathrm{H}_{45} \mathrm{NSiZr}$ : C, 65.30; H, 8.81; N, 2.72. Found: C, 65.13; H, 8.99; N, 2.63. mp 147-148 ${ }^{\circ} \mathrm{C}$. rac-(ebthi)Zr(NHCMe 3 )(cyclopentenyl) (1e). A light-orange solution of complex $1 a$ $(0.098 \mathrm{~g}, 0.222 \mathrm{mmol})$ in cyclopentene $(5.0 \mathrm{~mL})$ was heated in a glass vessel equipped with a vacuum stopcock under an atmosphere of $\mathrm{N}_{2}$ for $24 \mathrm{~h}$ at $75{ }^{\circ} \mathrm{C}$. The volatile materials were removed under vacuum to yield 1e as a dark brown oil $\left(0.102 \mathrm{~g}, 93 \%,>95 \%\right.$ pure by ${ }^{1} \mathrm{H}$ NMR spectroscopy (Figure S-4)), which was then crystallized at $-30{ }^{\circ} \mathrm{C}$ by slow diffusion of diethyl ether into toluene to yield analytically pure dark orange crystals. ${ }^{1} \mathrm{H}$ NMR $(500 \mathrm{MHz}) \delta 6.28(\mathrm{~d}$, $J=3.1 \mathrm{~Hz}, 1 \mathrm{H}, \mathrm{CpH}), 6.03(\mathrm{~d}, J=2.9 \mathrm{~Hz}, 1 \mathrm{H}, \mathrm{CpH}), 5.79(\mathrm{t}, J=2.0 \mathrm{~Hz}, 1 \mathrm{H}, \mathrm{ZrC}=\mathrm{CH}), 5.48(\mathrm{~d}$, 
$J=3.1 \mathrm{~Hz}, 1 \mathrm{H}, \mathrm{Cp} H), 5.29(\mathrm{~d}, J=2.9 \mathrm{~Hz}, 1 \mathrm{H}, \mathrm{Cp} H), 4.49$ (s, $1 \mathrm{H}, \mathrm{NH}), 2.8-2.7(\mathrm{~m}, 1 \mathrm{H}), 2.7-2.4$ (m, 8H), 2.4-2.3 (m, 6H), 2.3-2.2 (m, 1H), 1.9-1.8 (m, 2H), 1.8-1.6 (m, 4H), 1.5-1.4 (m, 4H), $\left.1.26(\mathrm{~s}, 9 \mathrm{H}, \mathrm{CMe})_{3}\right) \mathrm{ppm} .{ }^{13} \mathrm{C}\left\{{ }^{1} \mathrm{H}\right\}$ NMR $(125 \mathrm{MHz}) \delta 185.20(\mathrm{ZrC}=), 138.59(\mathrm{ZrC}=\mathrm{C}), 126.09$ $\left(\right.$ ebthi $\left.{ }_{\mathrm{q}}\right), 126.06\left(\mathrm{ebthi}_{\mathrm{q}}\right), 125.14\left(\mathrm{ebthi}_{\mathrm{q}}\right), 124.12\left(\mathrm{ebthi}_{\mathrm{q}}\right), 121.44\left(\mathrm{ebthi}_{\mathrm{q}}\right), 118.21\left(\mathrm{ebthi}_{\mathrm{q}}\right), 109.58$ (ebthi $), 105.77($ ebthi $), 105.15\left(\right.$ ebthi $\left._{\mathrm{t}}\right), 102.64\left(\mathrm{ebthi}_{\mathrm{t}}\right), 57.01\left(\mathrm{NCMe}_{3}\right), 46.76\left(\mathrm{CH}_{2}\right), 35.81$ $\left(\mathrm{CH}_{2}\right), 35.23\left(\mathrm{HNCMe}_{3}\right), 28.22\left(\mathrm{CH}_{2}\right), 27.62\left(\mathrm{CH}_{2}\right), 25.42\left(\mathrm{CH}_{2}\right), 24.97\left(\mathrm{CH}_{2}\right), 24.32\left(\mathrm{CH}_{2}\right)$, $23.86\left(\mathrm{CH}_{2}\right), 23.82\left(\mathrm{CH}_{2}\right), 23.50\left(\mathrm{CH}_{2}\right), 23.13\left(\mathrm{CH}_{2}\right), 22.73\left(\mathrm{CH}_{2}\right) \mathrm{ppm}$. Anal. Calcd for $\mathrm{C}_{29} \mathrm{H}_{41} \mathrm{NZr}$ : C, $70.38 ; \mathrm{H}, 8.35 ; \mathrm{N}, 2.83$. Found: $\mathrm{C}, 70.42 ; \mathrm{H}, 8.23 ; \mathrm{N}, 2.81$. mp $147{ }^{\circ} \mathrm{C}$.

rac-(ebthi)Zr(NHCMe 3 )(neohexenyl) (1f). A solution of complex 1a $(0.103 \mathrm{~g}, 0.232$ $\mathrm{mmol})$ in 3,3-dimethylbutene $(5.0 \mathrm{~mL})$ was heated in a glass vessel equipped with a vacuum stopcock under an atmosphere of $\mathrm{N}_{2}$ for $24 \mathrm{~h}$ at $75{ }^{\circ} \mathrm{C}$. The volatile materials were removed under vacuum to give a yellow oil. Diethyl ether was added to the oil and was subsequently removed under reduced pressure to yield $\mathbf{1 f}$ as a yellow solid $(0.109 \mathrm{~g}, 92 \%)$. Complex $\mathbf{1 f}$ is an analytically pure white crystalline solid upon crystallization from pentane at $-30^{\circ} \mathrm{C}(0.066 \mathrm{~g}$, $57 \%) .{ }^{1} \mathrm{H}$ NMR $(500 \mathrm{MHz}) \delta 6.66(\mathrm{~d}, J=18.6 \mathrm{~Hz}, 1 \mathrm{H}, \mathrm{ZrCH}=), 6.28(\mathrm{~d}, J=2.9 \mathrm{~Hz}, 1 \mathrm{H}, \mathrm{Cp} H)$, $6.09(\mathrm{~d}, J=18.6 \mathrm{~Hz}, 1 \mathrm{H}, \mathrm{ZrCH}=\mathrm{CH}), 6.04(\mathrm{~d}, J=2.9 \mathrm{~Hz}, 1 \mathrm{H}, \mathrm{Cp} H), 5.43(\mathrm{~d}, J=2.9 \mathrm{~Hz}, 1 \mathrm{H}$, $\mathrm{CpH}), 5.16(\mathrm{~d}, J=2.9 \mathrm{~Hz}, 1 \mathrm{H}, \mathrm{Cp} H), 4.72(\mathrm{~s}, 1 \mathrm{H}, \mathrm{NH}), 2.6-2.2(\mathrm{~m}, 12 \mathrm{H}), 1.9-1.8(\mathrm{~m}, 2 \mathrm{H}), 1.8-$ $1.6(\mathrm{~m}, 2 \mathrm{H}), 1.4-1.3(\mathrm{~m}, 4 \mathrm{H}), 1.26(\mathrm{~s}, 9 \mathrm{H}), 1.19(\mathrm{~s}, 9 \mathrm{H}) \mathrm{ppm} .{ }^{13} \mathrm{C}\left\{{ }^{1} \mathrm{H}\right\} \mathrm{NMR}(125 \mathrm{MHz}) \delta$ $166.48(\mathrm{ZrC}), 154.50(\mathrm{ZrCH}=C), 126.28\left(\mathrm{ebthi}_{\mathrm{q}}\right), 125.40\left(\mathrm{ebthi}_{\mathrm{q}}\right), 124.95\left(\mathrm{ebthi}_{\mathrm{q}}\right), 123.85$ $\left(\right.$ ebthi $\left.{ }_{\mathrm{q}}\right), 120.72\left(\right.$ ebthi $\left._{\mathrm{q}}\right), 117.83\left(\right.$ ebthi $\left._{\mathrm{q}}\right), 111.13\left(\mathrm{ebthi}_{\mathrm{t}}\right), 106.07\left(\right.$ ebthi $\left._{\mathrm{t}}\right), 105.31$ (ebthi $\left.\mathrm{i}_{\mathrm{t}}\right), 101.99$ (ebthi $\left.\left.\mathrm{i}_{\mathrm{t}}, 56.38\left(\mathrm{HNCMe}_{3}\right), 35.73(\mathrm{ZrCH}=\mathrm{CHCMe})_{3}\right), 34.97\left(\mathrm{HNCMe}_{3}\right), 30.08(\mathrm{ZrCH}=\mathrm{CHCMe})_{3}\right)$, $27.79\left(\mathrm{CH}_{2}\right), 27.66\left(\mathrm{CH}_{2}\right), 24.48\left(\mathrm{CH}_{2}\right), 24.45\left(\mathrm{CH}_{2}\right), 23.83\left(\mathrm{CH}_{2}\right), 23.61\left(\mathrm{CH}_{2}\right), 23.59\left(\mathrm{CH}_{2}\right)$, 23.17 $\left(\mathrm{CH}_{2}\right), 23.08\left(\mathrm{CH}_{2}\right) \mathrm{ppm}$. Anal. Calcd for $\mathrm{C}_{30} \mathrm{H}_{45} \mathrm{NZr}$ C, $70.53 ; \mathrm{H}, 8.88 ; \mathrm{N}, 2.74$. Found: $\mathrm{C}, 70.22 ; \mathrm{H}, 8.80 ; \mathrm{N}, 2.90$. mp $115^{\circ} \mathrm{C}$.

rac-(ebthi) Zr(NHCMe 3 )(1-hexenyl) (1g). A solution of complex 1a (0.095 g, 0.213 $\mathrm{mmol}$ ) in hexene $(5.0 \mathrm{~mL})$ was heated in a glass vessel equipped with a vacuum stopcock under an atmosphere of $\mathrm{N}_{2}$ for $24 \mathrm{~h}$ at $75^{\circ} \mathrm{C}$. The volatile materials were removed under vacuum to yield $1 \mathrm{~g}$ as yellow/orange powder $\left(0.108 \mathrm{~g}, 99 \%,>95 \%\right.$ pure by ${ }^{1} \mathrm{H}$ NMR spectroscopy (Figure S-5)). Purification of this complex has been hampered by its extreme solubility in pentane and other solvents. ${ }^{1} \mathrm{H}$ NMR $(500 \mathrm{MHz}) \delta 6.72(\mathrm{dt}, J=18.0 \mathrm{~Hz}, J=1.4 \mathrm{~Hz}, 1 \mathrm{H}, \mathrm{ZrCH}=), 6.27(\mathrm{~d}, J$ $=3.1 \mathrm{~Hz}, 1 \mathrm{H}, \mathrm{CpH}), 6.06(\mathrm{~d}, J=3.1 \mathrm{~Hz}, 1 \mathrm{H}, \mathrm{Cp} H), 5.96(\mathrm{dt}, J=18.0 \mathrm{~Hz}, J=6.1 \mathrm{~Hz}, 1 \mathrm{H}$, $\left.=\mathrm{CHC}_{4} \mathrm{H}_{9}\right), 5.41(\mathrm{~d}, J=3.1 \mathrm{~Hz}, 1 \mathrm{H}, \mathrm{Cp} H), 5.15(\mathrm{~d}, J=2.9 \mathrm{~Hz}, 1 \mathrm{H}, \mathrm{Cp} H), 4.73(\mathrm{~s}, 1 \mathrm{H}, \mathrm{NH}), 2.8-$ $2.7(\mathrm{~m}, 1 \mathrm{H}), 2.6-2.5(\mathrm{~m}, 5 \mathrm{H}), 2.5-2.4(\mathrm{~m}, 1 \mathrm{H}), 2.4-2.2(\mathrm{~m}, 7 \mathrm{H}), 1.9-1.8(\mathrm{~m}, 2 \mathrm{H}), 1.7-1.6(\mathrm{~m}, 2 \mathrm{H})$, 1.6-1.4 (m, 8H), $1.24\left(\mathrm{~s}, 9 \mathrm{H}, \mathrm{CMe}_{3}\right), 0.97\left(\mathrm{t}, J=7.3 \mathrm{~Hz}, 3 \mathrm{H}, \mathrm{CH}_{3}\right) \mathrm{ppm} .{ }^{13} \mathrm{C}\left\{{ }^{1} \mathrm{H}\right\} \mathrm{NMR}(125$ MHz) $\delta 175.73(\mathrm{ZrCH}=), 144.11(\mathrm{ZrCH}=C), 126.25\left(\mathrm{ebthi}_{\mathrm{q}}\right), 125.44\left(\mathrm{ebthi}_{\mathrm{q}}\right), 125.03\left(\mathrm{ebthi}_{\mathrm{q}}\right)$, $123.70\left(\right.$ ebthi $\left._{\mathrm{q}}\right), 120.85\left(\mathrm{ebthi}_{\mathrm{q}}\right), 117.95\left(\mathrm{ebthi}_{\mathrm{q}}\right), 110.68\left(\mathrm{ebthi}_{\mathrm{t}}\right), 106.12\left(\mathrm{ebthi}_{\mathrm{t}}\right), 105.18\left(\mathrm{ebthi}_{\mathrm{t}}\right)$, $101.76($ ebthi $), 56.33\left(\mathrm{HNCMe}_{3}\right), 40.45\left(\mathrm{CH}_{2}\right), 35.02(\mathrm{HNCMe}), 31.39\left(\mathrm{CH}_{2}\right), 27.73\left(\mathrm{CH}_{2}\right)$, $27.61\left(\mathrm{CH}_{2}\right), 24.66\left(\mathrm{CH}_{2}\right), 24.47\left(\mathrm{CH}_{2}\right), 23.81\left(\mathrm{CH}_{2}\right), 23.59\left(\mathrm{CH}_{2}\right), 23.56\left(\mathrm{CH}_{2}\right), 23.09\left(\mathrm{CH}_{2}\right)$, $22.87\left(\mathrm{CH}_{2}\right), 14.48\left(\mathrm{ZrCH}=\mathrm{CH}\left(\mathrm{CH}_{2}\right)_{3} \mathrm{CH}_{3}\right) \mathrm{ppm}$.

rac-(ebthi)Zr(NHCMe $)$ (mesityl) (1h). A solution of complex 1a $(0.100 \mathrm{~g}, 0.227$ mmol) in 1,3,5-trimethylbenzene $(3.5 \mathrm{~mL})$ and was heated in a glass vessel equipped with a vacuum stopcock under an atmosphere of $\mathrm{N}_{2}$ for $24 \mathrm{~h}$ at $75^{\circ} \mathrm{C}$. The volatile materials were removed under vacuum to yield $\mathbf{1 h}$ as a light brown solid. Due to the non-volatility of $1,3,5$ trimethylbenzene, this material includes $30 \mathrm{~mol} \%$ 1,3,5-trimethylbenzene as seen by ${ }^{1} \mathrm{H}$ NMR spectroscopy (calculated yield of $\mathbf{1 h}=96 \%$, otherwise $>95 \%$ pure by ${ }^{1} \mathrm{H}$ NMR spectroscopy (Figure S-6)). It was later found that when the light brown solid was washed quickly with 0.5 
$\mathrm{mL}$ of diethyl ether, a colorless powder remained, which was found to be analytically pure. ${ }^{1} \mathrm{H}$ NMR (500 MHz) $\delta 6.88(\mathrm{~s}, 2 \mathrm{H}, o-\operatorname{Ar} H), 6.53(\mathrm{~s}, 1 \mathrm{H}, p-\mathrm{Ar} H), 6.23(\mathrm{~d}, J=2.9 \mathrm{~Hz}, 1 \mathrm{H}, \mathrm{CpH}), 5.32$ $(\mathrm{d}, J=3.1 \mathrm{~Hz}, 1 \mathrm{H}, \mathrm{CpH}), 5.24(\mathrm{~d}, J=2.9 \mathrm{~Hz}, 1 \mathrm{H}, \mathrm{CpH}), 4.96(\mathrm{~d}, J=3.1 \mathrm{~Hz}, 1 \mathrm{H}, \mathrm{Cp} H), 4.89$ (s, $1 \mathrm{H}, \mathrm{NH}), 2.6-2.4,2.4-2.2(\mathrm{~m}, 11 \mathrm{H}), 2.30$ (s, 6H, m-ArMe), 2.15, $1.78\left(\mathrm{~m}, 5 \mathrm{H}, \mathrm{CH}_{2}\right.$, ebthi/ $\mathrm{ZrCH} \mathrm{H}_{2} \mathrm{Ar}$ ), 1.72 (d, $J=9.8 \mathrm{~Hz}, 1 \mathrm{H}, \mathrm{ZrCH}_{2} \mathrm{Ar}$ ), 1.7-1.4 (m, 5H) 1.33 (s, 9H, ZrNMe $e_{3}$ ppm. ${ }^{13} \mathrm{C}\left\{{ }^{1} \mathrm{H}\right\}$ NMR $(125 \mathrm{MHz}) \delta 155.88\left(\mathrm{ZrCH}_{2} \mathrm{C}\right), 136.48\left(\mathrm{~m}\right.$-Ar), 127.22 (ebthi $\left.\mathrm{q}_{\mathrm{q}}\right), 125.81\left(\mathrm{ebthi}_{\mathrm{q}}\right)$, $125.61\left(\mathrm{ebthi}_{\mathrm{q}}\right), 125.25(\mathrm{o}-\mathrm{Ar}), 125.08\left(\mathrm{ebthi}_{\mathrm{q}}\right), 121.76(p-\mathrm{Ar}), 120.37\left(\mathrm{ebthi}_{\mathrm{q}}\right), 118.56\left(\mathrm{ebthi}_{\mathrm{q}}\right)$, $112.32\left(\mathrm{ebthi}_{\mathrm{t}}\right), 106.75\left(\mathrm{ebthi}_{\mathrm{t}}\right), 106.24\left(\mathrm{ebthi}_{\mathrm{t}}\right), 102.61\left(\mathrm{ebthi}_{\mathrm{t}}\right), 56.28\left(\mathrm{HNCMe}_{3}\right), 51.44$ $\left(\mathrm{ZrCH}_{2}\right), 34.99\left(\mathrm{HNCMe}_{3}\right), 28.63\left(\mathrm{CH}_{2}\right), 27.73\left(\mathrm{CH}_{2}\right), 24.59\left(\mathrm{CH}_{2}\right), 24.26\left(\mathrm{CH}_{2}\right), 24.21\left(\mathrm{CH}_{2}\right)$, 23.67 $\left(\mathrm{CH}_{2}\right), 23.40\left(\mathrm{CH}_{2}\right), 23.04\left(\mathrm{CH}_{2}\right), 22.98\left(\mathrm{CH}_{2}\right), 21.85\left(\mathrm{Ar}-\mathrm{CH}_{3}\right) \mathrm{ppm}$. Anal. Calcd for $\mathrm{C}_{33} \mathrm{H}_{45} \mathrm{NZr}$ : C, 72.47; H, 8.29; N, 2.56. Found: C, 72.24; H, 8.30; N, 2.54.

rac-(ebthi)Zr(NHCMe 3 )(trans-2-phenylcyclopropyl) (1i). A solution of complex 1 a $(0.109 \mathrm{~g}, 0.245 \mathrm{mmol})$ in cyclopropylbenzene $(2.02 \mathrm{~g}, 17 \mathrm{mmol})$ and cyclohexane $(2.0 \mathrm{~mL})$ was heated in a glass vessel equipped with a vacuum stopcock under an atmosphere of $\mathrm{N}_{2}$ for $24 \mathrm{~h}$ at $75{ }^{\circ} \mathrm{C}$. The volatile materials were removed under vacuum to yield analytically pure $\mathbf{1 i}$ as a light red/orange foam $\left(0.108 \mathrm{~g}, 81 \%\right.$, >95\% pure by ${ }^{1} \mathrm{H}$ NMR spectroscopy (Figure S-7)). A 1:1 mixture of diastereomers was found by ${ }^{1} \mathrm{H}$ NMR spectroscopy, as can be seen by the duplication and equal intensity of signals for the protons $\alpha$ to the zirconium center (reported here as $0.5 \mathrm{H}$ for each proton), some of the ebthi $\mathrm{i}_{\mathrm{t}}$ resonances (reported here as $0.5 \mathrm{H}$ ), and the amide proton resonances (reported here as $0.5 \mathrm{H}$ ). All other signals are coincident for both of the diastereomers by ${ }^{1} \mathrm{H}$ NMR spectroscopy. Each of the signals in the ${ }^{13} \mathrm{C}\left\{{ }^{1} \mathrm{H}\right\}$ NMR are also seen in duplicate. The trans geometry of the zirconium atom and the phenyl ring was confirmed by 2D NOESY spectroscopy by the correlation seen between the phenyl protons and the proton alpha to the zirconium center. ${ }^{1} \mathrm{H}$ NMR $(400 \mathrm{MHz}) \delta$ 7.3-7.2 (m, 3H, Ar), 7.2-7.1 (m, 1H, Ar), 7.1-7.0 (m, $1 \mathrm{H}, \mathrm{Ar}), 6.28(\mathrm{~d}, J=3.3 \mathrm{~Hz}, 0.5 \mathrm{H}, \mathrm{CpH}), 6.22(\mathrm{t}, J=3.0 \mathrm{~Hz}, 1 \mathrm{H}, \mathrm{CpH}), 6.06(\mathrm{~d}, J=3.0 \mathrm{~Hz}$, $0.5 \mathrm{H}, \mathrm{CpH}), 5.37(\mathrm{~d}, J=2.8 \mathrm{~Hz}, 1 \mathrm{H}, \mathrm{CpH}), 5.22(\mathrm{~d}, J=3.0 \mathrm{~Hz}, 0.5 \mathrm{H}, \mathrm{CpH}), 5.02(\mathrm{~d}, J=3.0 \mathrm{~Hz}$, $0.5 \mathrm{H}, \mathrm{Cp} H), 4.83(\mathrm{~s}, 0.5 \mathrm{H}, \mathrm{NH}), 4.72(\mathrm{~s}, 0.5 \mathrm{H}, \mathrm{NH}), 3.2-3.1(\mathrm{~m}, 0.5 \mathrm{H}), 3.0-2.9(\mathrm{~m}, 0.5 \mathrm{H}), 2.6-2.4$ $(\mathrm{m}, 8 \mathrm{H}), 2.4-2.1(\mathrm{~m}, 6 \mathrm{H}), 2.1-1.9(\mathrm{~m}, 1 \mathrm{H}), 1.9-1.8(\mathrm{~m}, 1 \mathrm{H}), 1.5-1.4(\mathrm{~m}, 3 \mathrm{H}), 1.4-1.2(\mathrm{~m}, 2 \mathrm{H})$, $1.18\left(\mathrm{~s}, 9 \mathrm{H}, \mathrm{CMe} e_{3}\right), 0.9-0.6(\mathrm{~m}, 1 \mathrm{H}), 0.0{ }^{-} 0.1(\mathrm{~m}, 0.5 \mathrm{H}, \mathrm{ZrCH}),{ }^{-0}{ }^{-}{ }^{-0.2}(\mathrm{~m}, 0.5 \mathrm{H}, \mathrm{ZrCH}) \mathrm{ppm}$. ${ }^{13} \mathrm{C}\left\{{ }^{1} \mathrm{H}\right\}$ NMR $(100 \mathrm{MHz}) \delta 150.29\left(\mathrm{Ar}_{\mathrm{q}}\right), 149.78\left(\mathrm{Ar}_{\mathrm{q}}\right), 128.51\left(\mathrm{Ar}_{\mathrm{o}}\right), 128.31\left(\mathrm{Ar}_{\mathrm{o}}\right), 127.04$ $\left(\right.$ ebthi $\left._{\mathrm{q}}\right), 126.59\left(\mathrm{ebthi}_{\mathrm{q}}\right), 126.30\left(\mathrm{ebthi}_{\mathrm{q}}\right), 126.21\left(\mathrm{ebthi}_{\mathrm{q}}\right), 125.86\left(\mathrm{ebthi}_{\mathrm{q}}\right), 125.15\left(\mathrm{Ar}_{\mathrm{m}}\right), 124.95$ $\left(\mathrm{Ar}_{\mathrm{m}}\right), 124.52\left(\mathrm{ebthi}_{\mathrm{q}}\right), 124.32\left(\mathrm{Ar}_{\mathrm{p}}\right), 124.17\left(\mathrm{Ar}_{\mathrm{p}}\right), 123.59\left(\mathrm{ebthi}_{\mathrm{q}}\right), 122.89\left(\mathrm{ebthi}_{\mathrm{q}}\right), 120.57$ $\left(\mathrm{ebthi}_{\mathrm{q}}\right), 120.41\left(\mathrm{ebthi}_{\mathrm{q}}\right), 118.38\left(\mathrm{ebthi}_{\mathrm{q}}\right), 118.31\left(\mathrm{ebthi}_{\mathrm{q}}\right), 113.52\left(\mathrm{ebthi}_{\mathrm{t}}\right), 112.81\left(\mathrm{ebthi}_{\mathrm{t}}\right), 105.81$ $\left(\right.$ ebthi $\left.\mathrm{t}_{\mathrm{t}}\right), 105.76\left(\mathrm{ebthi}_{\mathrm{t}}\right), 105.73\left(\mathrm{ebthi}_{\mathrm{t}}\right), 105.53\left(\mathrm{ebthi}_{\mathrm{t}}\right), 102.06\left(\mathrm{ebthi}_{\mathrm{t}}\right), 101.84\left(\mathrm{ebthi}_{\mathrm{t}}\right), 56.67$ $\left(\mathrm{HNCMe}_{3}\right), 56.59\left(\mathrm{HNCMe}_{3}\right), 43.94(\mathrm{ZrCH}), 42.64(\mathrm{ZrCH}), 35.14\left(\mathrm{HNCMe}_{3}\right), 35.06\left(\mathrm{HNCMe}_{3}\right)$, $\left.31.59{ }^{c}{ }^{\mathrm{Pr}} \mathrm{CH}_{2}\right), 29.87\left({ }^{c \mathrm{Pr}} \mathrm{CH}_{2}\right), 27.85\left(\mathrm{CH}_{2}\right), 27.79\left(\mathrm{CH}_{2}\right), 27.64\left(\mathrm{CH}_{2}\right), 27.55\left(\mathrm{CH}_{2}\right), 27.21$ $\left(\mathrm{CH}_{2}\right), 26.55\left(\mathrm{CH}_{2}\right), 24.65\left(\mathrm{CH}_{2}\right), 24.60\left(\mathrm{CH}_{2}\right), 24.48\left(\mathrm{CH}_{2}\right), 24.42\left(\mathrm{CH}_{2}\right), 23.99\left(\mathrm{CH}_{2}\right), 23.91$ $\left(\mathrm{CH}_{2}\right), 23.74\left(\mathrm{CH}_{2}\right), 23.59\left(\mathrm{CH}_{2}\right), 23.54\left(\mathrm{CH}_{2}\right), 23.48\left(\mathrm{CH}_{2}\right), 23.32\left(\mathrm{CH}_{2}\right), 23.27\left(\mathrm{CH}_{2}\right), 23.21$ $\left(\mathrm{CH}_{2}\right), 23.11\left(\mathrm{CH}_{2}\right)$ ppm.

rac-(ebthi)Zr(NHCMe $\left.\mathbf{N}_{3}\right)($ neohexyl) (1j). A solution of complex 1a $(0.096 \mathrm{~g}, 0.22$ mmol) in 2,2-dimethylbutane $(4.0 \mathrm{~mL})$ was heated in a glass vessel equipped with a vacuum stopcock under an atmosphere of $\mathrm{N}_{2}$ for $24 \mathrm{~h}$ at $75{ }^{\circ} \mathrm{C}$. The volatile materials were removed under vacuum to yield $\mathbf{1 j}$ as a yellow foam $\left(0.108 \mathrm{~g}, 98 \%,>95 \%\right.$ pure by ${ }^{1} \mathrm{H}$ NMR spectroscopy (Figure S-8)). Purification of this complex has been hampered by its extreme solubility in pentane and other solvents. ${ }^{1} \mathrm{H}$ NMR $(500 \mathrm{MHz}) \delta 6.16(\mathrm{~d}, J=2.9 \mathrm{~Hz}, 1 \mathrm{H}, \mathrm{CpH}), 6.09(\mathrm{~d}, J=$ $3.1 \mathrm{~Hz}, 1 \mathrm{H}, \mathrm{CpH}), 5.38(\mathrm{~d}, J=3.1 \mathrm{~Hz}, 1 \mathrm{H}, \mathrm{CpH}), 5.17(\mathrm{~d}, J=2.9 \mathrm{~Hz}, 1 \mathrm{H}, \mathrm{CpH}), 4.79$ (s, 1H, 
$\mathrm{NH}), 2.9-2.0(\mathrm{~m}, 11 \mathrm{H}), 2.0-1.3(\mathrm{~m}, 11 \mathrm{H}), 1.21(\mathrm{~s}, 9 \mathrm{H}), 1.11(\mathrm{~s}, 9 \mathrm{H}), 0.56(\mathrm{td}, J=12.4 \mathrm{~Hz}, J=3.1$ $\left.\mathrm{Hz}, 1 \mathrm{H}, \mathrm{ZrCH}_{2}\right), 0.27\left(\mathrm{td}, J=12.8 \mathrm{~Hz}, J=4.2 \mathrm{~Hz}, 1 \mathrm{H}, \mathrm{ZrCH}_{2}\right) \mathrm{ppm} .{ }^{13} \mathrm{C}\left\{{ }^{1} \mathrm{H}\right\} \mathrm{NMR}(125 \mathrm{MHz})$ $\delta 126.74\left(\mathrm{ebthi}_{\mathrm{q}}\right), 124.88\left(\mathrm{ebthi}_{\mathrm{q}}\right), 123.29\left(\mathrm{ebthi}_{\mathrm{q}}\right), 123.16\left(\mathrm{ebthi}_{\mathrm{q}}\right), 119.92\left(\mathrm{ebthi}_{\mathrm{q}}\right), 118.68$ $\left(\mathrm{ebthi}_{\mathrm{q}}\right), 109.71\left(\mathrm{ebthi}_{\mathrm{t}}\right), 106.00\left(\mathrm{ebthi}_{\mathrm{t}}\right), 105.72\left(\mathrm{ebthi}_{\mathrm{t}}\right), 101.29\left(\mathrm{ebthi}_{\mathrm{t}}\right), 55.79\left(\mathrm{HNCMe}_{3}\right), 51.65$ $\left(\mathrm{ZrCH}_{2}\right), 38.21\left(\mathrm{CH}_{2}\right), 34.78\left(\mathrm{HNCMe}_{3}\right), 33.50\left(\mathrm{Zr}\left(\mathrm{CH}_{2}\right)_{2} \mathrm{CMe}_{3}\right), 29.70\left(\mathrm{Zr}\left(\mathrm{CH}_{2}\right)_{2} \mathrm{CMe}_{3}\right), 28.15$ $\left(\mathrm{CH}_{2}\right), 27.04\left(\mathrm{CH}_{2}\right), 25.59\left(\mathrm{CH}_{2}\right), 24.68\left(\mathrm{CH}_{2}\right), 24.63\left(\mathrm{CH}_{2}\right), 23.87\left(\mathrm{CH}_{2}\right), 23.59\left(\mathrm{CH}_{2}\right), 23.49$ $\left(\mathrm{CH}_{2}\right), 23.48\left(\mathrm{CH}_{2}\right), 23.26\left(\mathrm{CH}_{2}\right), 23.23\left(\mathrm{CH}_{2}\right) \mathrm{ppm}$.

rac-(ebthi)Zr(NHCMe $)$ (4-methylpentyl) (1k). A solution of complex 1a (0.092 g, $0.21 \mathrm{mmol})$ in 2-methylpentane $(4.8 \mathrm{~mL})$ was heated in a glass vessel equipped with a vacuum stopcock under an atmosphere of $\mathrm{N}_{2}$ for $24 \mathrm{~h}$ at $75{ }^{\circ} \mathrm{C}$. The volatile materials were removed under vacuum to yield $1 \mathbf{k}$ as a yellow oil $\left(0.099 \mathrm{~g}, 92 \%,>95 \%\right.$ pure by ${ }^{1} \mathrm{H}$ NMR spectroscopy (Figure S-9)). Purification of this complex has been hampered by its extreme solubility in pentane and other solvents. ${ }^{1} \mathrm{H}$ NMR $(500 \mathrm{MHz}) \delta 6.12(\mathrm{~d}, J=2.9 \mathrm{~Hz}, 1 \mathrm{H}, \mathrm{CpH}), 6.10(\mathrm{~d}, J=$ $3.1 \mathrm{~Hz}, 1 \mathrm{H}, \mathrm{CpH}), 5.40(\mathrm{~d}, J=2.9 \mathrm{~Hz}, 1 \mathrm{H}, \mathrm{CpH}), 5.14(\mathrm{~d}, J=3.1 \mathrm{~Hz}, 1 \mathrm{H}, \mathrm{CpH}), 4.78(\mathrm{~s}, 1 \mathrm{H}$, $\mathrm{NH}), 2.8-2.7(\mathrm{~m}, 2 \mathrm{H}), 2.7-2.6(\mathrm{~m}, 1 \mathrm{H}), 2.6-2.4(\mathrm{~m}, 7 \mathrm{H}), 2.4-2.3(\mathrm{~m}, 4 \mathrm{H}), 2.2-2.1(\mathrm{~m}, 1 \mathrm{H}), 2.0-1.9$ $(\mathrm{m}, 1 \mathrm{H}), 1.9-1.6(\mathrm{~m}, 6 \mathrm{H}), 1.6-1.5(\mathrm{~m}, 1 \mathrm{H}), 1.5-1.4(\mathrm{~m}, 5 \mathrm{H}), 1.23(\mathrm{~s}, 9 \mathrm{H}, \mathrm{CMe} 3), 1.06(\mathrm{dd}, J=6.6$ $\left.\mathrm{Hz}, J=1.2 \mathrm{~Hz}, 6 \mathrm{H}, \mathrm{CH}_{3}\right), 0.54\left(\mathrm{td}, J=11.9 \mathrm{~Hz}, J=4.1 \mathrm{~Hz}, 1 \mathrm{H}, \mathrm{ZrCH}_{2} \mathrm{C}_{5} \mathrm{H}_{11}\right), 0.40(\mathrm{td}, J=12.1$ $\left.\mathrm{Hz}, J=4.7 \mathrm{~Hz}, 1 \mathrm{H}, \mathrm{ZrCH}_{2} \mathrm{C}_{5} \mathrm{H}_{11}\right) \mathrm{ppm} .{ }^{13} \mathrm{C}\left\{{ }^{1} \mathrm{H}\right\} \mathrm{NMR}(125 \mathrm{MHz}) \delta 126.61$ (ebthi $\left.\mathrm{q}_{\mathrm{q}}\right), 124.81$ $\left(\right.$ ebthi $\left.\mathrm{q}_{\mathrm{q}}\right), 123.19\left(\mathrm{ebthi}_{\mathrm{q}}\right), 123.01\left(\mathrm{ebthi}_{\mathrm{q}}\right), 119.91\left(\mathrm{ebthi}_{\mathrm{q}}\right), 118.80\left(\mathrm{ebthi}_{\mathrm{q}}\right), 109.71\left(\mathrm{ebthi}_{\mathrm{t}}\right), 105.87$ (ebthi $), 105.51\left(\right.$ ebthi $\left._{\mathrm{t}}\right), 101.22\left(\mathrm{ebthi}_{\mathrm{t}}\right), 55.69\left(\mathrm{HNCMe}_{3}\right), 48.17\left(\mathrm{ZrCH}_{2} \mathrm{C}_{4} \mathrm{H}_{9}\right), 45.29\left(\mathrm{CH}_{2}\right)$, $34.81\left(\mathrm{HNCMe}_{3}\right), 28.34\left(\mathrm{Zr}\left(\mathrm{CH}_{2}\right)_{3} \mathrm{CH}\right), 28.00\left(\mathrm{CH}_{2}\right), 27.10\left(\mathrm{CH}_{2}\right), 24.66\left(\mathrm{CH}_{2}\right), 24.55\left(\mathrm{CH}_{2}\right)$, $23.76\left(\mathrm{CH}_{2}\right), 23.56\left(\mathrm{CH}_{2}\right), 23.52\left(\mathrm{CH}_{2}\right), 23.48\left(\mathrm{Zr}\left(\mathrm{CH}_{2}\right)_{3} \mathrm{CH}\left(\mathrm{CH}_{3}\right) M e_{\mathrm{a}}\right), 23.44\left(\mathrm{CH}_{2}\right), 23.21$ $\left(\mathrm{Zr}\left(\mathrm{CH}_{2}\right)_{3} \mathrm{CH}\left(\mathrm{CH}_{3}\right) M e_{\mathrm{b}}\right), 23.26\left(\mathrm{CH}_{2}\right), 23.21\left(\mathrm{CH}_{2}\right) \mathrm{ppm}$.

rac-(ebthi)Zr(NHCMe 3 )(3-methylpentyl) (11). A solution of complex 1a $(0.100 \mathrm{~g}$, $0.225 \mathrm{mmol})$ in 3 -methylpentane $(3.5 \mathrm{~mL})$ and cyclohexane $(0.50 \mathrm{~mL})$ was heated in a glass vessel equipped with a vacuum stopcock under an atmosphere of $\mathrm{N}_{2}$ for $24 \mathrm{~h}$ at $75{ }^{\circ} \mathrm{C}$. The volatile materials were removed under vacuum to yield $\mathbf{1 l}$ as a yellow oil $(0.105 \mathrm{~g}, 90 \%,>95 \%$ pure by ${ }^{1} \mathrm{H}$ NMR spectroscopy (Figure S-10)). Purification of this complex has been hampered by its extreme solubility in pentane and other solvents. A 1:1 mixture of diastereomers was found by ${ }^{1} \mathrm{H}$ NMR spectroscopy, as can be seen by the duplication and equal intensity of signals for the protons $\alpha$ to the zirconium center (reported here as $0.5 \mathrm{H}$ for each proton) and from both of the alkyl methyl groups (reported here as $1.5 \mathrm{H}$ ). All other signals are coincident for both of the diastereomers by ${ }^{1} \mathrm{H}$ NMR spectroscopy. Most of the signals in the ${ }^{13} \mathrm{C}\left\{{ }^{1} \mathrm{H}\right\}$ NMR spectrum are also seen in duplicate. ${ }^{1} \mathrm{H} \mathrm{HMR}(500 \mathrm{MHz}) \delta 6.13(\mathrm{t}, J=2.8 \mathrm{~Hz}, 1 \mathrm{H}, \mathrm{CpH}), 6.09(\mathrm{t}, J=3.7$ $\mathrm{Hz}, 1 \mathrm{H}, \mathrm{CpH}), 5.39$ (d, J=1.8 Hz, 1H, CpH), 5.14 (t, J=3.2 Hz, 1H, CpH), 4.77 (d, J = 4.3 Hz, $1 \mathrm{H}, \mathrm{NH}), 2.8-2.3(\mathrm{~m}, 12 \mathrm{H}), 2.2-2.1(\mathrm{~m}, 1 \mathrm{H}), 1.9-1.5(\mathrm{~m}, 8 \mathrm{H}), 1.5-1.4(\mathrm{~m}, 3 \mathrm{H}), 1.4-1.3(\mathrm{~m}, 4 \mathrm{H})$, $1.20\left(\mathrm{~s}, 9 \mathrm{H}, \mathrm{CMe}_{3}\right), 1.12\left(\mathrm{~d}, J=6.4 \mathrm{~Hz}, 1.5 \mathrm{H}, \mathrm{ZrCH}_{2} \mathrm{CHCH}_{3}\right), 1.10(\mathrm{~d}, J=6.4 \mathrm{~Hz}, 1.5 \mathrm{H}$, $\left.\mathrm{ZrCH}_{2} \mathrm{CHCH}_{3}\right), 1.06\left(\mathrm{t}, \mathrm{J}=7.4 \mathrm{~Hz}, 1.5 \mathrm{H}, \mathrm{ZrCH}_{2} \mathrm{CH}\left(\mathrm{CH}_{3}\right) \mathrm{CH}_{2} \mathrm{CH}_{3}\right), 1.04$ (t, J = 7.6 Hz, 1.5H, $\left.\mathrm{ZrCH}_{2} \mathrm{CH}\left(\mathrm{CH}_{3}\right) \mathrm{CH}_{2} \mathrm{CH}_{3}\right), 0.60\left(\mathrm{td}, J=12.4 \mathrm{~Hz}, J=3.5 \mathrm{~Hz}, 0.5 \mathrm{H}, \mathrm{ZrCH}_{2} \mathrm{C}_{5} \mathrm{H}_{11}\right), 0.47(\mathrm{td}, J=$ $\left.12.4 \mathrm{~Hz}, J=3.5 \mathrm{~Hz}, 0.5 \mathrm{H}, \mathrm{ZrCH}_{2} \mathrm{C}_{5} \mathrm{H}_{11}\right), 0.39\left(\mathrm{td}, J=11.6 \mathrm{~Hz}, J=4.9 \mathrm{~Hz}, 0.5 \mathrm{H}, \mathrm{ZrCH}_{2} \mathrm{C}_{5} \mathrm{H}_{11}\right)$, $0.27\left(\mathrm{td}, J=12.4 \mathrm{~Hz}, J=4.1 \mathrm{~Hz}, 0.5 \mathrm{H}, \mathrm{ZrCH}_{2} \mathrm{C}_{5} \mathrm{H}_{11}\right) \mathrm{ppm} .{ }^{13} \mathrm{C}\left\{{ }^{1} \mathrm{H}\right\} \mathrm{NMR}(125 \mathrm{MHz}) \delta 126.67$ $\left(\right.$ ebthi $\left.\mathrm{q}_{\mathrm{q}}\right), 126.64\left(\mathrm{ebthi}_{\mathrm{q}}\right), 124.83\left(\mathrm{ebthi}_{\mathrm{q}}\right), 124.80\left(\mathrm{ebthi}_{\mathrm{q}}\right), 123.20\left(\mathrm{ebthi}_{\mathrm{q}}\right), 123.03\left(\mathrm{ebthi}_{\mathrm{q}}\right), 123.02$ $\left(\right.$ ebthi $\left.\mathrm{q}_{\mathrm{q}}\right), 119.91\left(\mathrm{ebthi}_{\mathrm{q}}\right), 118.76\left(\mathrm{ebthi}_{\mathrm{q}}\right), 118.73\left(\mathrm{ebthi}_{\mathrm{q}}\right), 109.83\left(\mathrm{ebthi}_{\mathrm{t}}\right), 109.73\left(\mathrm{ebthi}_{\mathrm{t}}\right), 105.94$ $\left(\mathrm{ebthi}_{\mathrm{t}}\right), 105.89\left(\mathrm{ebthi}_{\mathrm{t}}\right), 105.59\left(\mathrm{ebthi}_{\mathrm{t}}\right), 105.57\left(\mathrm{ebthi}_{\mathrm{t}}\right), 101.22\left(\mathrm{ebthi}_{\mathrm{t}}\right), 101.21$ (ebthi $\left._{\mathrm{t}}\right), 55.75$ $\left(\mathrm{HNCMe}_{3}\right), 55.70\left(\mathrm{HNCMe}_{3}\right), 43.93\left(\mathrm{ZrCH}_{2}\right), 43.82\left(\mathrm{ZrCH}_{2}\right), 41.55\left(\mathrm{Zr}\left(\mathrm{CH}_{2}\right)_{2} \mathrm{CH}\right), 41.50$ $\left(\mathrm{Zr}\left(\mathrm{CH}_{2}\right)_{2} \mathrm{CH}\right), 34.80\left(\mathrm{HNCMe}_{3}\right), 29.92\left(\mathrm{CH}_{2}\right), 29.73\left(\mathrm{CH}_{2}\right), 28.06\left(\mathrm{CH}_{2}\right), 28.04\left(\mathrm{CH}_{2}\right), 27.07$ 
$\left(\mathrm{CH}_{2}\right), 24.69\left(\mathrm{CH}_{2}\right), 24.68\left(\mathrm{CH}_{2}\right), 24.58\left(\mathrm{CH}_{2}\right), 23.80\left(\mathrm{CH}_{2}\right), 23.57\left(\mathrm{CH}_{2}\right), 23.51\left(\mathrm{CH}_{2}\right), 23.49$ $\left(\mathrm{CH}_{2}\right), 23.48\left(\mathrm{CH}_{2}\right), 23.27\left(\mathrm{CH}_{2}\right), 23.22\left(\mathrm{CH}_{2}\right), 23.21\left(\mathrm{CH}_{2}\right), 19.70\left(\mathrm{Zr}\left(\mathrm{CH}_{2}\right)_{2} \mathrm{CHCH}_{3}\right), 19.69$ $\left(\mathrm{Zr}\left(\mathrm{CH}_{2}\right)_{2} \mathrm{CHCH}_{3}\right), 12.37\left(\mathrm{Zr}\left(\mathrm{CH}_{2}\right)_{2} \mathrm{CH}\left(\mathrm{CH}_{3}\right) \mathrm{CH}_{2} \mathrm{CH}_{3}\right), 12.37\left(\mathrm{Zr}\left(\mathrm{CH}_{2}\right)_{2} \mathrm{CH}\left(\mathrm{CH}_{3}\right) \mathrm{CH}_{2} \mathrm{CH}_{3}\right) \mathrm{ppm}$.

rac-(ebthi)Zr(NHCMe 3 )(phenyl) (1m). A solution of complex 1a $(0.094 \mathrm{~g}, 0.21 \mathrm{mmol})$ in benzene $(5.0 \mathrm{~mL})$ was heated in a glass vessel equipped with a vacuum stopcock under an atmosphere of $\mathrm{N}_{2}$ for $24 \mathrm{~h}$ at $75{ }^{\circ} \mathrm{C}$. The volatile materials were removed under vacuum. The resulting yellow oil was subsequently dissolved in diethyl ether $(4 \mathrm{~mL})$, and the ether was then removed under vacuum to yield $\mathbf{1 m}$ as a colorless foam $\left(0.100 \mathrm{~g}, 93 \%,>95 \%\right.$ pure by ${ }^{1} \mathrm{H}$ NMR spectroscopy (Figure S-11)). The foam was washed with $n$-pentane $(1 \mathrm{~mL})$ and the remaining powder was dried under reduced pressure, leaving $1 \mathrm{~m}$ as an analytically pure, colorless solid. ${ }^{1} \mathrm{H}$ NMR $(500 \mathrm{MHz}) \delta 7.79\left(\mathrm{~d}, J=6.7 \mathrm{~Hz}, 2 \mathrm{H}, \operatorname{Ar} H_{\mathrm{o}}\right), 7.26\left(\mathrm{dd}, J=7.3, J=6.7 \mathrm{~Hz}, 2 \mathrm{H}, \operatorname{Ar} H_{\mathrm{m}}\right)$, $7.15\left(\mathrm{t}, J=7.3 \mathrm{~Hz}, 1 \mathrm{H}, \operatorname{Ar} H_{\mathrm{p}}\right), 6.45(\mathrm{~d}, J=2.8 \mathrm{~Hz}, 1 \mathrm{H}, \mathrm{Cp} H), 6.06(\mathrm{~d}, J=3.1 \mathrm{~Hz}, 1 \mathrm{H}, \mathrm{Cp} H)$, $5.50(\mathrm{~d}, J=2.9 \mathrm{~Hz}, 1 \mathrm{H}, \mathrm{Cp} H), 5.31(\mathrm{~d}, J=2.9 \mathrm{~Hz}, 1 \mathrm{H}, \mathrm{Cp} H), 4.76(\mathrm{~s}, 1 \mathrm{H}, \mathrm{NH}), 2.6-2.5(\mathrm{~m}, 4 \mathrm{H})$, 2.4-2.2 (m, 7H), 2.2-2.1 (m, 1H), 1.8-1.6 (m, 2H), 1.5-1.4 (m, 2H), 1.33 (s, 9H, CMe 3 ), 1.2-1.1 (m, 2H), 1.1-0.9 (m, 2H) ppm. ${ }^{13} \mathrm{C}\left\{{ }^{1} \mathrm{H}\right\}$ NMR (125 MHz) $\delta 182.33(\mathrm{ZrC}), 141.88(\mathrm{Ar}), 126.73$ (ebthi $\left.{ }_{q}\right), 126.31$ (Ar), $126.26\left(\right.$ ebthi $\left._{q}\right), 125.56\left(\right.$ ebthi $\left._{q}\right), 124.76$ (Ar), 123.52 (ebthi $), 122.35$ (ebthi $\left.)_{q}\right), 118.93\left(\right.$ ebthi $\left._{q}\right), 112.73\left(\right.$ ebthi $\left._{t}\right), 107.09\left(\right.$ ebthi $\left._{t}\right), 105.08\left(\right.$ ebthi $\left._{t}\right), 102.07\left(\right.$ ebthi $\left._{t}\right), 57.38$ $\left(\mathrm{HNCMe}_{3}\right), 35.06\left(\mathrm{HNCMe}_{3}\right), 27.72\left(\mathrm{CH}_{2}\right), 27.12\left(\mathrm{CH}_{2}\right), 24.66\left(\mathrm{CH}_{2}\right), 24.01\left(\mathrm{CH}_{2}\right), 23.54$ $\left(\mathrm{CH}_{2}\right), 23.31\left(\mathrm{CH}_{2}\right), 23.26\left(\mathrm{CH}_{2}\right), 23.08\left(\mathrm{CH}_{2}\right), 22.49\left(\mathrm{CH}_{2}\right), 21.75\left(\mathrm{CH}_{2}\right) \mathrm{ppm}$. Anal. Calcd for $\mathrm{C}_{30} \mathrm{H}_{39} \mathrm{NZr}$ : C, 71.37; H, 7.79; N, 2.77. Found: $\mathrm{C}, 71.03 ; \mathrm{H}, 7.64 ; \mathrm{N}, 3.11$. mp 151-152 ${ }^{\circ} \mathrm{C}$.

rac-(ebthi) $\mathrm{Zr}$ (NHCMe 3 ) (3,5-bis(trifluoromethyl)phenyl) (1n). A solution of complex 1a $(0.108 \mathrm{~g}, 0.244 \mathrm{mmol})$ in 1,3-bis(trifluoromethyl)benzene $(1.84 \mathrm{~g}, 8.60 \mathrm{mmol})$ and cyclohexane $(2.0 \mathrm{~mL})$ was heated in a glass vessel equipped with a vacuum stopcock under an atmosphere of $\mathrm{N}_{2}$ for $24 \mathrm{~h}$ at $75{ }^{\circ} \mathrm{C}$. The volatile materials were removed under vacuum to leave 1n as a yellow solid. Complex 1n was obtained as an analytically pure light yellow foam after dissolving in benzene and removing volatile materials under vacuum $(0.127 \mathrm{~g}, 81 \%)$. Although some ${ }^{13} \mathrm{C}$ resonances are reported here, not all ${ }^{13} \mathrm{C}$ resonances were evidenced in the ${ }^{13} \mathrm{C}\left\{{ }^{1} \mathrm{H}\right\}$ NMR spectrum due to ${ }^{13} \mathrm{C}-{ }^{19} \mathrm{~F}$ coupling under standard pulse sequences. ${ }^{1} \mathrm{H}$ NMR (400 MHz) $\delta$ $8.32\left(\mathrm{~s}, 2 \mathrm{H}, \operatorname{Ar} H_{\mathrm{o}}\right), 7.72\left(\mathrm{~s}, 1 \mathrm{H}, \operatorname{Ar} H_{\mathrm{p}}\right), 6.38(\mathrm{~d}, J=2.5 \mathrm{~Hz}, 1 \mathrm{H}, \mathrm{Cp} H), 5.70(\mathrm{~d}, J=2.5 \mathrm{~Hz}, 1 \mathrm{H}$, $\mathrm{CpH}), 5.40(\mathrm{~d}, J=2.8 \mathrm{~Hz}, 1 \mathrm{H}, \mathrm{CpH}), 5.16(\mathrm{~d}, J=2.5 \mathrm{~Hz}, 1 \mathrm{H}, \mathrm{CpH}), 4.99$ (s, $1 \mathrm{H}, \mathrm{NH}), 2.7-2.6$ (m, $1 \mathrm{H}), 2.6-2.4(\mathrm{~m}, 4 \mathrm{H}), 2.3-2.1(\mathrm{~m}, 4 \mathrm{H}), 2.1-1.9(\mathrm{~m}, 2 \mathrm{H}), 1.7-1.5(\mathrm{~m}, 2 \mathrm{H}), 1.4-1.3(\mathrm{~m}, 2 \mathrm{H})$, $1.28\left(\mathrm{~s}, 9 \mathrm{H}, \mathrm{CMe}_{3}\right), 1.19(\mathrm{~s}, 1 \mathrm{H}), 1.1-1.0(\mathrm{~m}, 1 \mathrm{H}), 1.0-0.9(\mathrm{~m}, 1 \mathrm{H}), 0.8-0.7(\mathrm{~m}, 1 \mathrm{H}), 0.6-0.4(\mathrm{~m}$, 1H) ppm. ${ }^{13} \mathrm{C}\left\{{ }^{1} \mathrm{H}\right\}$ NMR $(100 \mathrm{MHz}) \delta 185.01(\mathrm{~s}, \mathrm{ZrC}), 141.46\left(\mathrm{~s}, \mathrm{Ar}_{\mathrm{o}}\right), 128.12$ (s, ebthi $\left.{ }_{\mathrm{q}}\right)$, $127.63\left(\mathrm{~s}\right.$, ebthi $\left._{\mathrm{q}}\right), 126.78\left(\mathrm{~s}, \mathrm{ebthi}_{\mathrm{q}}\right), 126.28\left(\mathrm{~s}\right.$, ebthi $\left._{\mathrm{q}}\right), 124.04\left(\mathrm{~s}, \mathrm{ebthi}_{\mathrm{q}}\right), 119.65\left(\mathrm{~s}, \mathrm{ebthi}_{\mathrm{q}}\right.$ ), 118.4-118.1 (m, Ar $\left.\mathrm{m}_{\mathrm{m}}\right), 111.96\left(\mathrm{~s}, \mathrm{ebthi}_{\mathrm{t}}\right), 107.56(\mathrm{~s}$, ebthi $), 104.93\left(\mathrm{~s}\right.$, ebthi $\left._{\mathrm{t}}\right), 102.90\left(\mathrm{~s}, \mathrm{ebthi}_{\mathrm{t}}\right)$, 57.89 (s, $\mathrm{HNCMe}_{3}$ ), 34.80 (s, $\mathrm{HNCMe}_{3}$ ), 27.72 (s, $\mathrm{CH}_{2}$ ), 27.05 (s, $\mathrm{CH}_{2}$ ), 24.39 (s, $\mathrm{CH}_{2}$ ), 23.91 (s, $\mathrm{CH}_{2}$ ), 23.45 (s, $\mathrm{CH}_{2}$ ), 23.24 (s, $\mathrm{CH}_{2}$ ), 23.10 (s, $\mathrm{CH}_{2}$ ), 22.83 (s, $\left.\mathrm{CH}_{2}\right), 22.17$ (s, $\left.\mathrm{CH}_{2}\right), 21.56$ (s, $\mathrm{CH}_{2}$ ) ppm. ${ }^{19} \mathrm{~F}$ NMR $\left(377 \mathrm{MHz}, \mathrm{C}_{6} \mathrm{D}_{6}\right) \delta 61.92$ (s) ppm. Anal. Calcd for $\mathrm{C}_{32} \mathrm{H}_{37} \mathrm{~F}_{6} \mathrm{NZr}: \mathrm{C}$, 59.97; H, 5.82; N, 2.19. Found: C, 60.10; H, 6.02; N, 2.02 .

rac-(ebthi) $\mathrm{Zr}$ (NHCMe $)$ (3,5-diisopropylphenyl) (10). A solution of complex $1 \mathrm{a}(0.112$ $\mathrm{g}, 0.252 \mathrm{mmol})$ in 1,3-diisopropylbenzene $(1.95 \mathrm{~g}, 12.0 \mathrm{mmol})$ and cyclohexane $(2.0 \mathrm{~mL})$ was heated in a glass vessel equipped with a vacuum stopcock under an atmosphere of $\mathrm{N}_{2}$ for $24 \mathrm{~h}$ at $75^{\circ} \mathrm{C}$. The volatile materials were removed under vacuum. Analytically pure complex 10 was obtained as a yellow solid after dissolving in benzene and removing volatile materials under vacuum $(0.101 \mathrm{~g}, 68 \%) .{ }^{1} \mathrm{H}$ NMR $(400 \mathrm{MHz}) \delta 7.51\left(\mathrm{~s}, 2 \mathrm{H}, \operatorname{Ar} H_{\mathrm{o}}\right), 6.85\left(\mathrm{~s}, 1 \mathrm{H}, \operatorname{Ar} H_{\mathrm{p}}\right), 6.52(\mathrm{~d}, J$ $=3.3 \mathrm{~Hz}, 1 \mathrm{H}, \mathrm{Cp} H), 6.15(\mathrm{~d}, J=3.0 \mathrm{~Hz}, 1 \mathrm{H}, \mathrm{Cp} H), 5.50(\mathrm{~d}, J=3.0 \mathrm{~Hz}, 1 \mathrm{H}, \mathrm{Cp} H), 5.41(\mathrm{~d}, J=$ 
$3.3 \mathrm{~Hz}, 1 \mathrm{H}, \mathrm{CpH}), 4.72(\mathrm{~s}, 1 \mathrm{H}, \mathrm{NH}), 3.0-2.8$ (m, 2H), 2.8-2.7 (m, 4H), 2.7-2.5 (m, 5H), 2.4-2.2 $(\mathrm{m}, 5 \mathrm{H}), 2.2-2.1(\mathrm{~m}, 2 \mathrm{H}), 1.8-1.6(\mathrm{~m}, 2 \mathrm{H}), 1.41(\mathrm{~s}, 9 \mathrm{H}, \mathrm{CMe}), 1.38$ (dd, J = 7.1 Hz, J = 1.3 Hz, $\left.6 \mathrm{H},{ }^{i \mathrm{Pr}} \mathrm{Me}\right), 0.9-0.8(\mathrm{~m}, 1 \mathrm{H}), 0.7-0.6(\mathrm{~m}, 1 \mathrm{H}) \mathrm{ppm} .{ }^{13} \mathrm{C}\left\{{ }^{1} \mathrm{H}\right\}$ NMR $(100 \mathrm{MHz}) \delta 182.04(\mathrm{ZrC})$, $145.29\left(\mathrm{Ar}_{\mathrm{m}}\right), 137.37\left(\mathrm{Ar}_{\mathrm{p}}\right), 126.33\left(\mathrm{ebthi}_{\mathrm{q}}\right), 125.82\left(\mathrm{ebthi}_{\mathrm{q}}\right), 125.31\left(\mathrm{ebthi}_{\mathrm{q}}\right), 122.81\left(\mathrm{ebthi}_{\mathrm{q}}\right)$, $122.39\left(\mathrm{ebthi}_{\mathrm{q}}\right), 121.34\left(\mathrm{Ar}_{\mathrm{o}}\right), 119.04\left(\mathrm{ebthi}_{\mathrm{q}}\right), 113.14\left(\mathrm{ebthi}_{\mathrm{t}}\right), 107.36\left(\mathrm{ebthi}_{\mathrm{t}}\right), 104.48\left(\mathrm{ebthi}_{\mathrm{t}}\right)$, $101.51\left(\mathrm{ebthi}_{\mathrm{t}}\right), 57.32\left(\mathrm{HNCMe}_{3}\right), 35.35\left({ }^{i \mathrm{Pr}} \mathrm{CH}\right), 35.24(\mathrm{HNCMe}), 27.49\left(\mathrm{CH}_{2}\right), 26.84\left(\mathrm{CH}_{2}\right)$, $25.05\left({ }^{i \mathrm{Pr}} \mathrm{Me}\right), 24.88\left({ }^{i \mathrm{Pr}} \mathrm{Me}\right), 24.60\left(\mathrm{CH}_{2}\right), 23.99\left(\mathrm{CH}_{2}\right), 23.51\left(\mathrm{CH}_{2}\right), 23.27\left(\mathrm{CH}_{2}\right), 23.15\left(\mathrm{CH}_{2}\right)$, $22.36\left(\mathrm{CH}_{2}\right), 21.56\left(\mathrm{CH}_{2}\right)$ ppm. Anal. Calcd for $\mathrm{C}_{36} \mathrm{H}_{51} \mathrm{NZr}: \mathrm{C}, 73.41 ; \mathrm{H}, 8.73 ; \mathrm{N}, 2.38$. Found: C, 73.03; H, 8.56; N, 2.30.

rac $-(\mathbf{e b t h i}) \mathrm{Zr}\left(\mathrm{NCMe}_{3}\right)(\mathbf{p y r})(3)$. A solution of complex $1 \mathbf{a}(0.094 \mathrm{~g}, 0.21 \mathrm{mmol})$ and pyridine $(0.025 \mathrm{~mL}, 0.32 \mathrm{mmol})$ in pentane $(5.0 \mathrm{~mL})$ was heated at $75{ }^{\circ} \mathrm{C}$ in a glass vessel equipped with a vacuum stopcock for $24 \mathrm{~h}$ under an atmosphere of $\mathrm{N}_{2}$. Analytically pure orange, needle-like crystals formed in the vessel equipped with a vacuum stopcock over the course of the reaction. The pentane solution was removed from the crystals with a pipette in the glovebox, and the remaining volatile materials were removed under reduced pressure to yield $4(0.072 \mathrm{~g}, 67 \%)$. ${ }^{1} \mathrm{H}$ NMR $(500 \mathrm{MHz}) \delta 8.93(\mathrm{dd}, J=4.9 \mathrm{~Hz}, J=1.7 \mathrm{~Hz}, 2 \mathrm{H}, \mathrm{Zr}-\mathrm{Py}$ ), $6.94(\mathrm{~d}, J=3.2 \mathrm{~Hz}, 1 \mathrm{H}$, $\mathrm{CpH}), 6.75$ (tt, $\left.J=7.6 \mathrm{~Hz}, J=1.7 \mathrm{~Hz}, 1 \mathrm{H}, \mathrm{Zr}-\mathrm{Py})_{\mathrm{p}}\right), 6.5-6.4(\mathrm{~m}, 1 \mathrm{H}, \mathrm{Zr}-\mathrm{Py}$ ), 5.76 (d, $J=3.1 \mathrm{~Hz}$, $1 \mathrm{H}, \mathrm{CpH}), 5.46(\mathrm{~d}, J=2.4 \mathrm{~Hz}, 1 \mathrm{H}, \mathrm{CpH}), 5.35(\mathrm{~d}, J=2.6 \mathrm{~Hz}, 1 \mathrm{H}, \mathrm{CpH}), 3.8-3.7(\mathrm{~m}, 1 \mathrm{H}), 2.9-$ $2.5(\mathrm{~m}, 8 \mathrm{H}), 2.4-2.1(\mathrm{~m}, 4 \mathrm{H}), 2.0-1.9(\mathrm{~m}, 1 \mathrm{H}), 1.9-1.8(\mathrm{~m}, 1 \mathrm{H}), 1.8-1.6(\mathrm{~m}, 1 \mathrm{H}), 1.3-1.0(\mathrm{~m}, 2 \mathrm{H})$, $1.48\left(\mathrm{~s}, 9 \mathrm{H}, \mathrm{CMe} e_{3}\right), 0.8-0.6(\mathrm{~m}, 1 \mathrm{H}), 0.4-0.2(\mathrm{~m}, 1 \mathrm{H}) \mathrm{ppm} .{ }^{13} \mathrm{C}\left\{{ }^{1} \mathrm{H}\right\} \mathrm{NMR}(125 \mathrm{MHz}) \delta 154.88$ $\left(\mathrm{Zr}-\mathrm{Py} \mathrm{y}_{\mathrm{o}}\right), 137.73\left(\mathrm{Zr}-\mathrm{Py} \mathrm{y}_{\mathrm{m}}\right), 127.33\left(\mathrm{ebthi}_{\mathrm{q}}\right), 126.02\left(\mathrm{ebthi}_{\mathrm{q}}\right), 123.91\left(\mathrm{ebthi}_{\mathrm{q}}\right), 123.58\left(\mathrm{Zr}-\mathrm{Py} \mathrm{p}_{\mathrm{p}}\right)$ $118.91\left(\mathrm{ebthi}_{\mathrm{q}}\right), 117.98\left(\mathrm{ebthi}_{\mathrm{q}}\right), 113.56\left(\mathrm{ebthi}_{\mathrm{q}}\right), 108.82\left(\mathrm{ebthi}_{\mathrm{t}}\right), 106.01\left(\mathrm{ebthi}_{\mathrm{t}}\right), 103.72\left(\mathrm{ebthi}_{\mathrm{t}}\right)$, $99.10\left(\mathrm{ebthi}_{\mathrm{t}}\right), 62.26\left(\mathrm{HNCMe}_{3}\right), 35.66\left(\mathrm{HNCMe}_{3}\right), 27.06\left(\mathrm{CH}_{2}\right), 26.20\left(\mathrm{CH}_{2}\right), 25.41\left(\mathrm{CH}_{2}\right)$, $25.12\left(\mathrm{CH}_{2}\right), 24.61\left(\mathrm{CH}_{2}\right), 24.49\left(\mathrm{CH}_{2}\right), 24.12\left(\mathrm{CH}_{2}\right), 23.52\left(\mathrm{CH}_{2}\right), 23.42\left(\mathrm{CH}_{2}\right), 23.19\left(\mathrm{CH}_{2}\right)$ ppm. Anal. Calcd for $\mathrm{C}_{29} \mathrm{H}_{38} \mathrm{~N}_{2} \mathrm{Zr}$ : C, 68.86; H, 7.57; N, 5.54. Found: C, 68.52; H, 7.42; N, 5.40 .

rac-(ebthi) $\mathbf{Z r F}\left(\mathbf{C}_{9} \mathbf{H}_{9} \mathbf{N}_{2} \mathbf{F}_{4}\right)$ (4). A solution of pentafluoropyridine $(0.40 \mathrm{~mL}, 3.6 \mathrm{mmol})$ and complex 1a $(0.102 \mathrm{~g}, 0.231 \mathrm{mmol})$ in cyclohexane $(4.0 \mathrm{~mL})$ was heated in a glass vessel equipped with a vacuum stopcock under an atmosphere of $\mathrm{N}_{2}$ for $40 \mathrm{~h}$ at $75{ }^{\circ} \mathrm{C}$. The volatile materials were removed under vacuum to yield $\mathbf{5}$ as a light orange foam. The foam dissolved readily in benzene $(4.0 \mathrm{~mL})$, which was subsequently removed under vacuum to yield a colorless powder $\left(0.136 \mathrm{~g}, 99 \%,>95 \%\right.$ pure by ${ }^{1} \mathrm{H}$ NMR spectroscopy (Figure S-12)). Complex 5 was crystallized at $-30{ }^{\circ} \mathrm{C}$ by slow diffusion of pentane into diethyl ether to yield yellow, needle-like crystals in the centric orthorhombic space group Pbca with one molecule in the asymmetric unit. Although some ${ }^{13} \mathrm{C}$ resonances are reported here, not all ${ }^{13} \mathrm{C}$ resonances were evidenced in the ${ }^{13} \mathrm{C}\left\{{ }^{1} \mathrm{H}\right\}$ NMR spectrum due to multiple-bond ${ }^{13} \mathrm{C}-{ }^{19} \mathrm{~F}$ coupling under standard pulse sequences. ${ }^{1} \mathrm{H}$ NMR $\left(500 \mathrm{MHz}, d_{8}\right.$-THF) $\delta 5.85(\mathrm{t}, J=3.3 \mathrm{~Hz}, 1 \mathrm{H}, \mathrm{Cp} H), 5.64(\mathrm{t}, J=3.0 \mathrm{~Hz}, 1 \mathrm{H}, \mathrm{Cp} H)$, 5.43 (br s, $1 \mathrm{H}, \mathrm{CpH}), 5.30(\mathrm{~d}, J=2.8 \mathrm{~Hz}, 1 \mathrm{H}, \mathrm{CpH}), 3.28(\mathrm{td}, J=13.3 \mathrm{~Hz}, J=6.2 \mathrm{~Hz}, 1 \mathrm{H}), 3.10$ $(\mathrm{td}, J=13.7 \mathrm{~Hz}, J=6.2 \mathrm{~Hz}, 1 \mathrm{H}), 3.0-2.8(\mathrm{~m}, 4 \mathrm{H}), 2.7-2.6(\mathrm{~m}, 1 \mathrm{H}), 2.6-2.5(\mathrm{~m}, 2 \mathrm{H}), 2.5-2.4(\mathrm{~m}$, $1 \mathrm{H}), 2.4-2.2(\mathrm{~m}, 4 \mathrm{H}), 2.0-1.9(\mathrm{~m}, 2 \mathrm{H}), 1.8-1.7(\mathrm{~m}, 1 \mathrm{H}), 1.7-1.6(\mathrm{~m}, 1 \mathrm{H}), 1.6-1.4(\mathrm{~m}, 2 \mathrm{H}), 1.33(\mathrm{~d}$, $\left.J=1.8 \mathrm{~Hz}, 9 \mathrm{H}, \mathrm{NCMe} e_{3}\right) \mathrm{ppm} .{ }^{13} \mathrm{C}\left\{{ }^{1} \mathrm{H}\right\} \mathrm{NMR}(125 \mathrm{MHz}) \delta 131.58\left(\mathrm{~s}, \mathrm{ebthi}_{\mathrm{q}}\right), 131.09$ (br s, ebthi $\left._{\mathrm{q}}\right), 129.25\left(\mathrm{~d}, J=3.1 \mathrm{~Hz}\right.$, ebthi $\left._{\mathrm{q}}\right), 128.95\left(\mathrm{~d}, J=3.3 \mathrm{~Hz}\right.$, ebthi $\left._{\mathrm{q}}\right), 128.25\left(\mathrm{~s}\right.$, ebthi $\left._{\mathrm{q}}\right), 123.53(\mathrm{~d}$, $J=1.7 \mathrm{~Hz}$, ebthi $\left._{\mathrm{q}}\right), 119.31\left(\mathrm{~d}, J=4.0 \mathrm{~Hz}\right.$, ebthi $\left._{\mathrm{t}}\right), 114.57\left(\mathrm{~s}, \mathrm{ebthi}_{\mathrm{t}}\right), 106.28(\mathrm{~d}, J=2.1 \mathrm{~Hz}$, ebthi $\left.\mathrm{t}_{\mathrm{t}}\right), 98.74\left(\mathrm{dd}, J=8.2 \mathrm{~Hz}, J=1.4 \mathrm{~Hz}\right.$, ebthi $\left._{\mathrm{t}}\right), 55.44\left(\mathrm{~s}, \mathrm{HNCMe}_{3}\right), 32.63(\mathrm{dd}, J=10.1 \mathrm{~Hz}, J=$ $6.1 \mathrm{~Hz}, \mathrm{HNCMe}$ ), 27.25 (s, $\left.\mathrm{CH}_{2}\right), 27.16\left(\mathrm{~s}, \mathrm{CH}_{2}\right), 25.85\left(\mathrm{~s}, \mathrm{CH}_{2}\right), 25.02\left(\mathrm{~s}, \mathrm{CH}_{2}\right), 24.11\left(\mathrm{~s}, \mathrm{CH}_{2}\right)$, $23.72\left(\mathrm{~s}, \mathrm{CH}_{2}\right), 23.7-23.5\left(\mathrm{~m}, \mathrm{CH}_{2}\right), 22.83$ (d, $\left.J=2.8 \mathrm{~Hz}, \mathrm{CH}_{2}\right) \mathrm{ppm} .{ }^{19} \mathrm{~F}$ NMR $\left(377 \mathrm{MHz}, \mathrm{C}_{6} \mathrm{D}_{6}\right)$ 
$\delta 15.08(\mathrm{~s}),{ }^{-9} 97.9(\mathrm{~m}),{ }^{-1} 39.3(\mathrm{~m}),{ }^{-156.9}(\mathrm{br} \mathrm{s}),{ }^{-187.4}(\mathrm{~m}) \mathrm{ppm}$. Anal. Calcd for $\mathrm{C}_{29} \mathrm{H}_{33} \mathrm{~F}_{5} \mathrm{~N}_{2} \mathrm{Zr}$ : C, 58.46; H, 5.58; N, 4.70. Found: C, 58.51; H, 5.52; N, 4.58. 


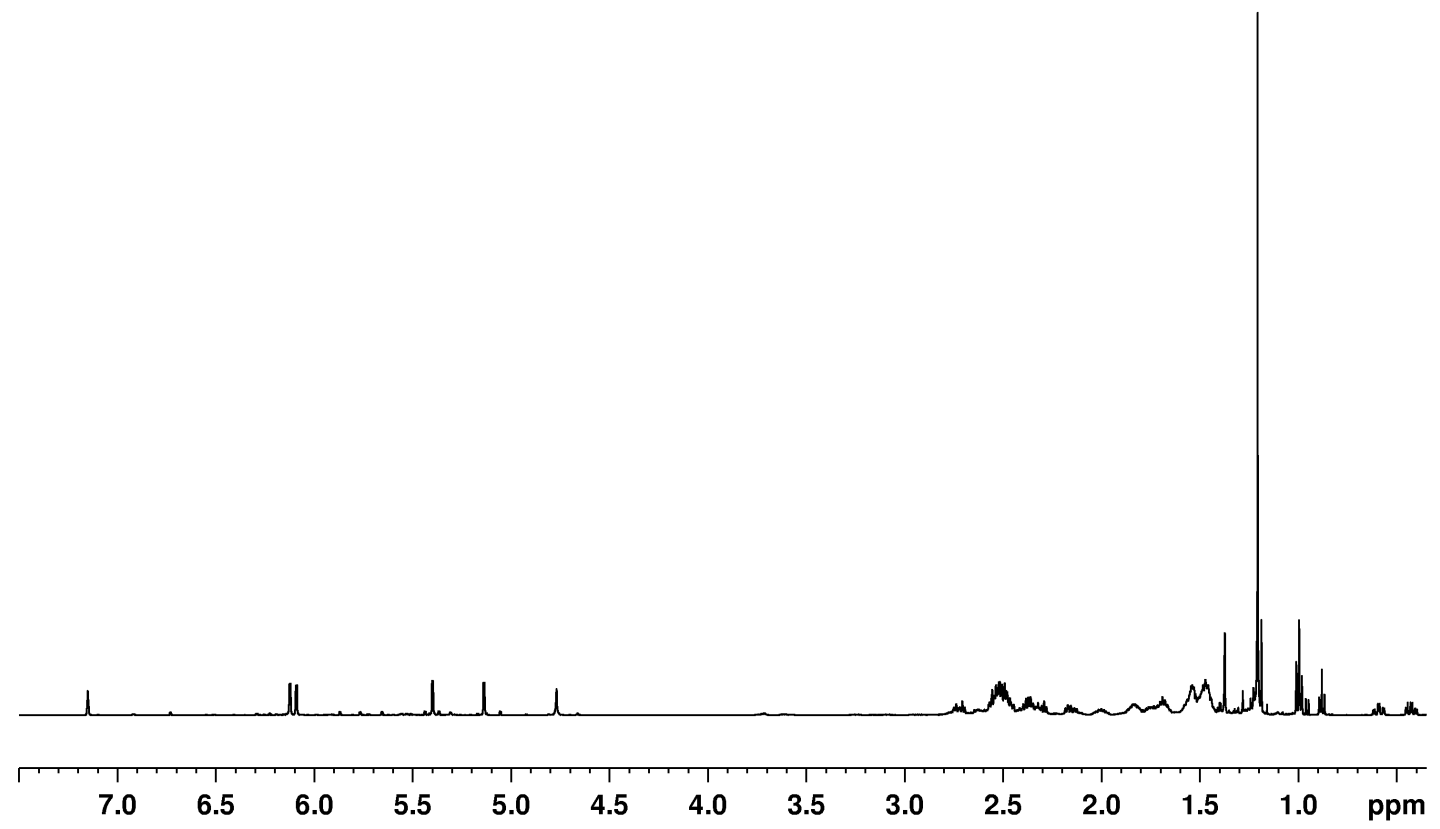

Figure S-1. ${ }^{1} \mathrm{H}$ NMR spectrum of isolated complex $\mathbf{1 b}$.

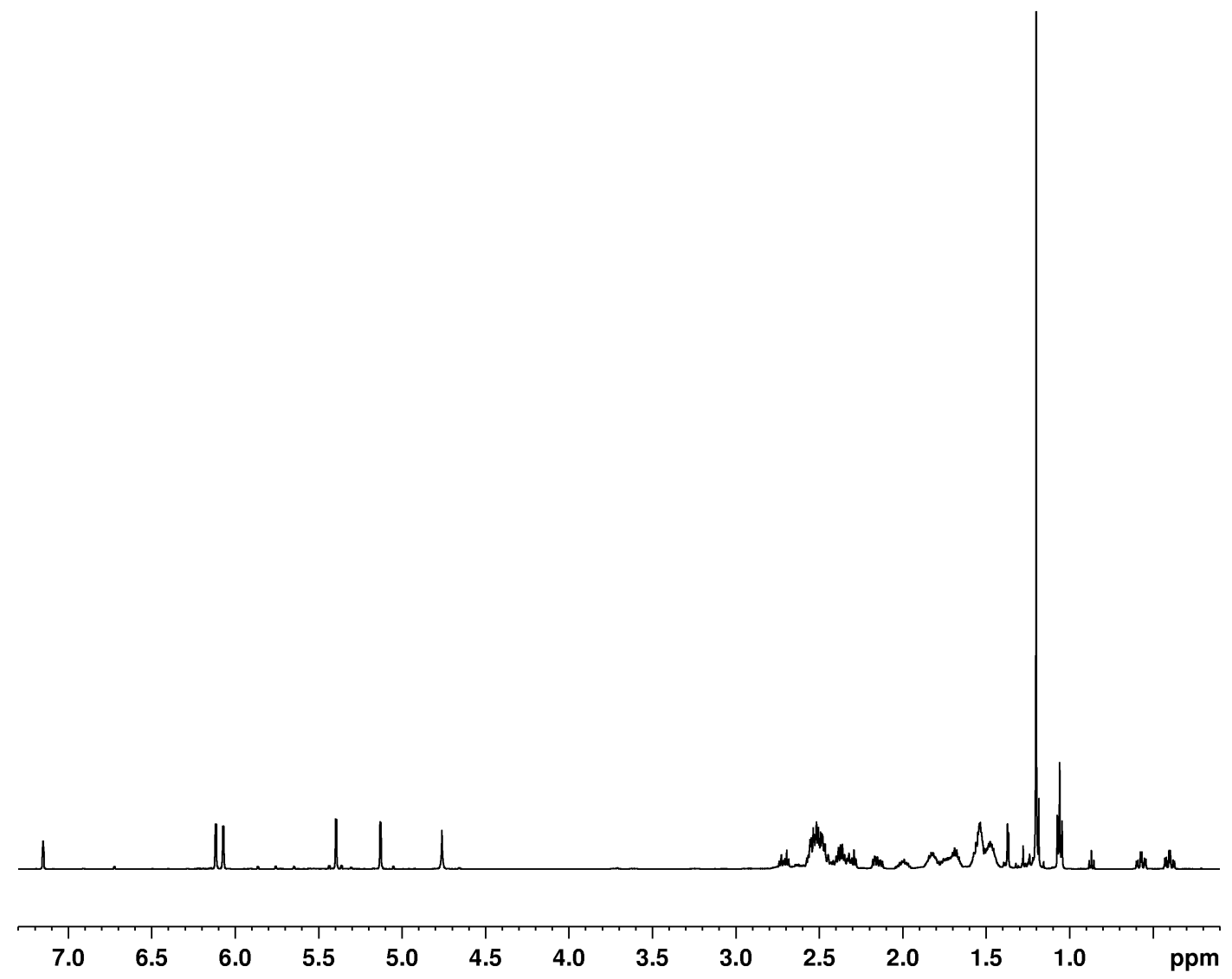

Figure S-2. ${ }^{1} \mathrm{H}$ NMR spectrum of isolated complex 1c. 


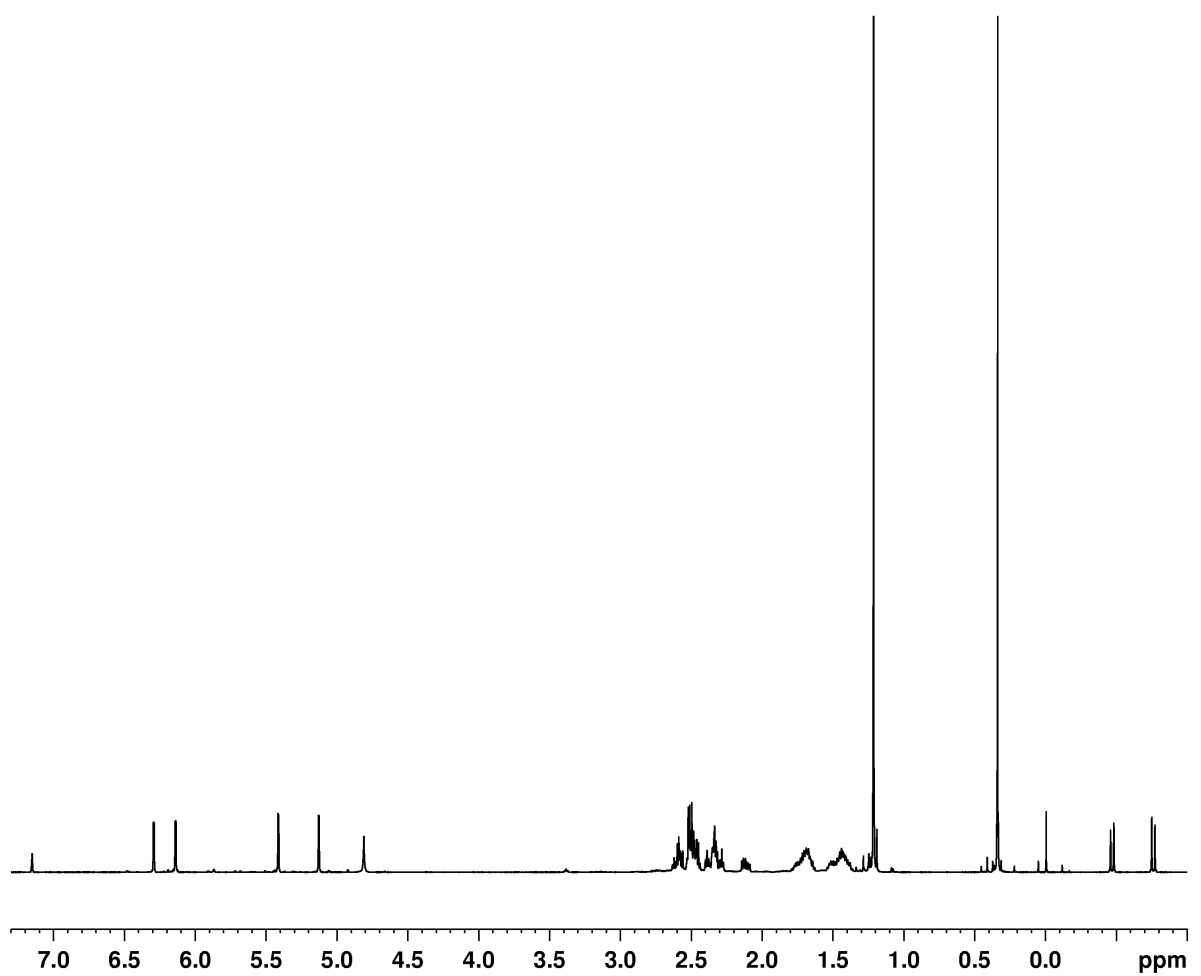

Figure S-3. ${ }^{1} \mathrm{H}$ NMR spectrum of isolated complex $\mathbf{1 d}$.

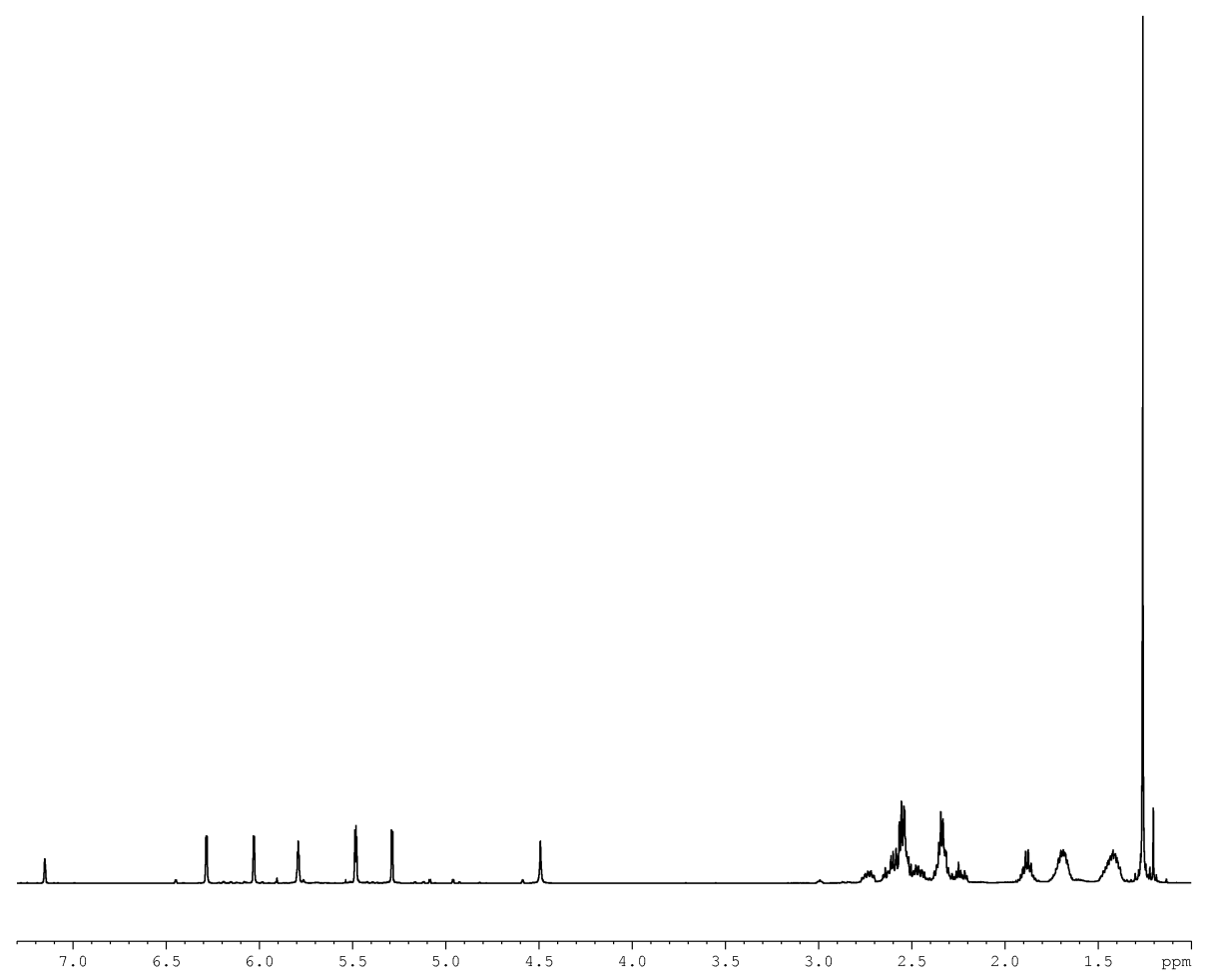

Figure S-4. ${ }^{1}$ H NMR spectrum of isolated complex $1 e$. 


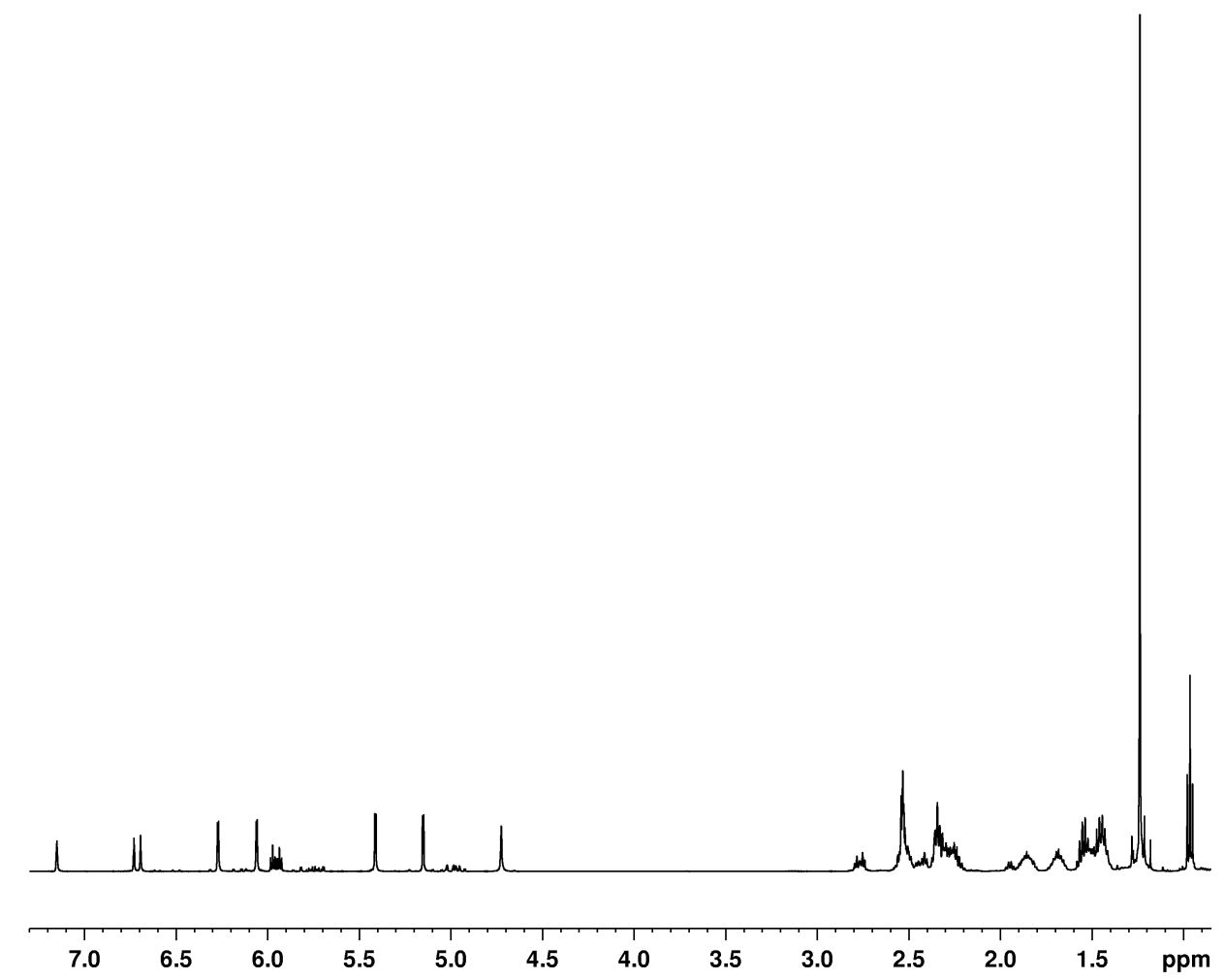

Figure S-5. ${ }^{1} \mathrm{H}$ NMR spectrum of isolated complex $\mathbf{1 g}$.

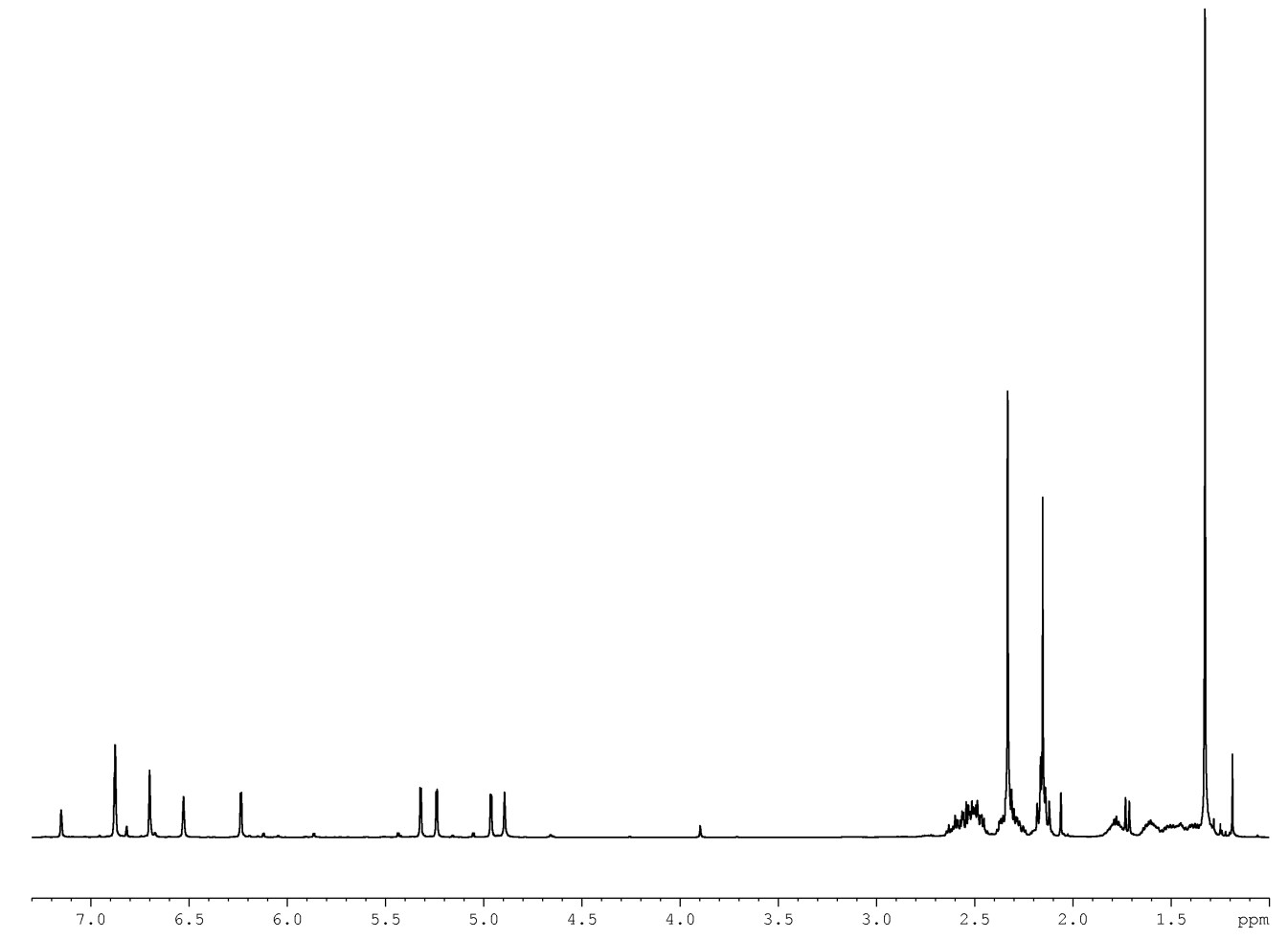

Figure S-6. ${ }^{1}$ H NMR spectrum of isolated complex $\mathbf{1 h}$. 


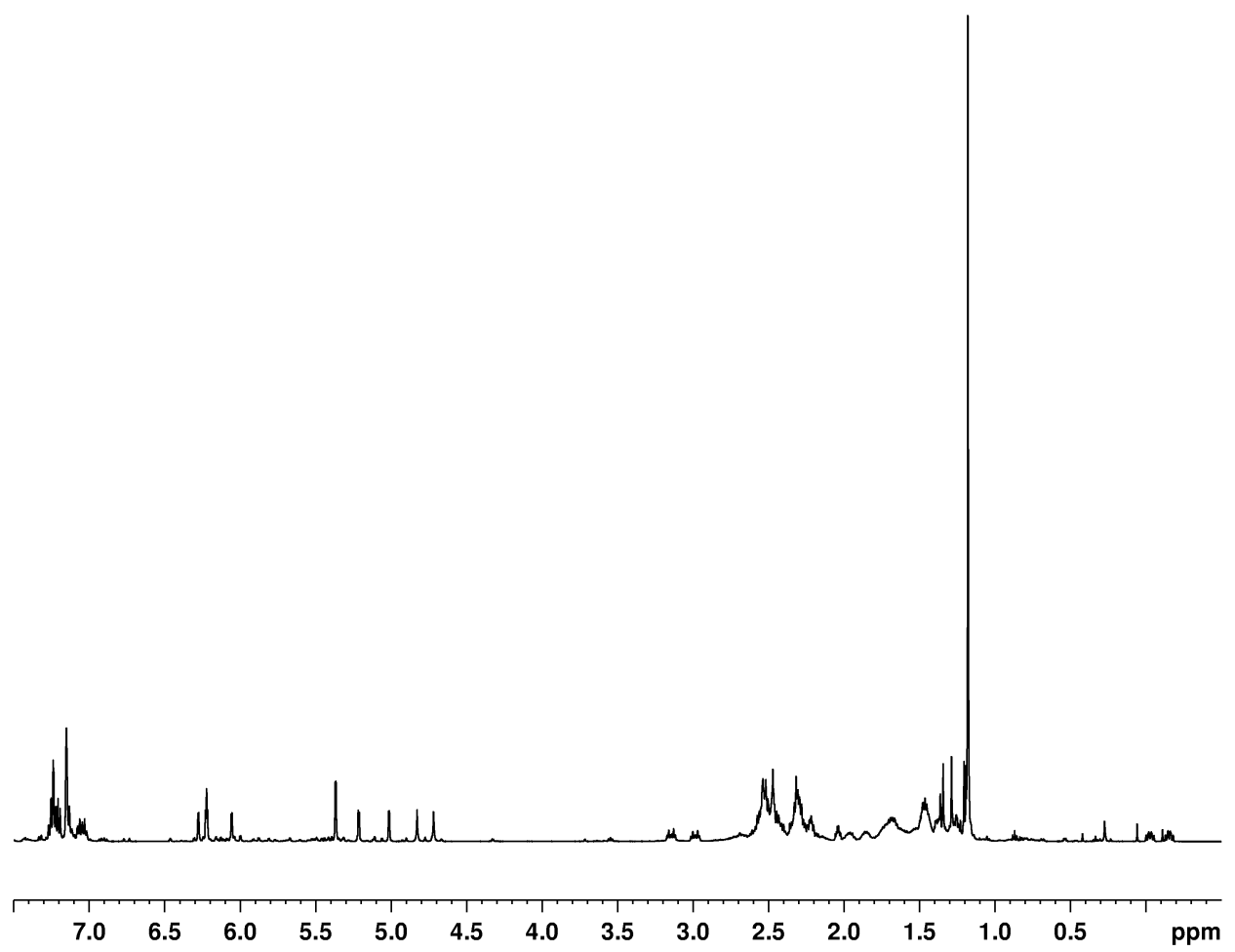

Figure S-7. ${ }^{1} \mathrm{H}$ NMR spectrum of isolated complex $\mathbf{1 i}$.

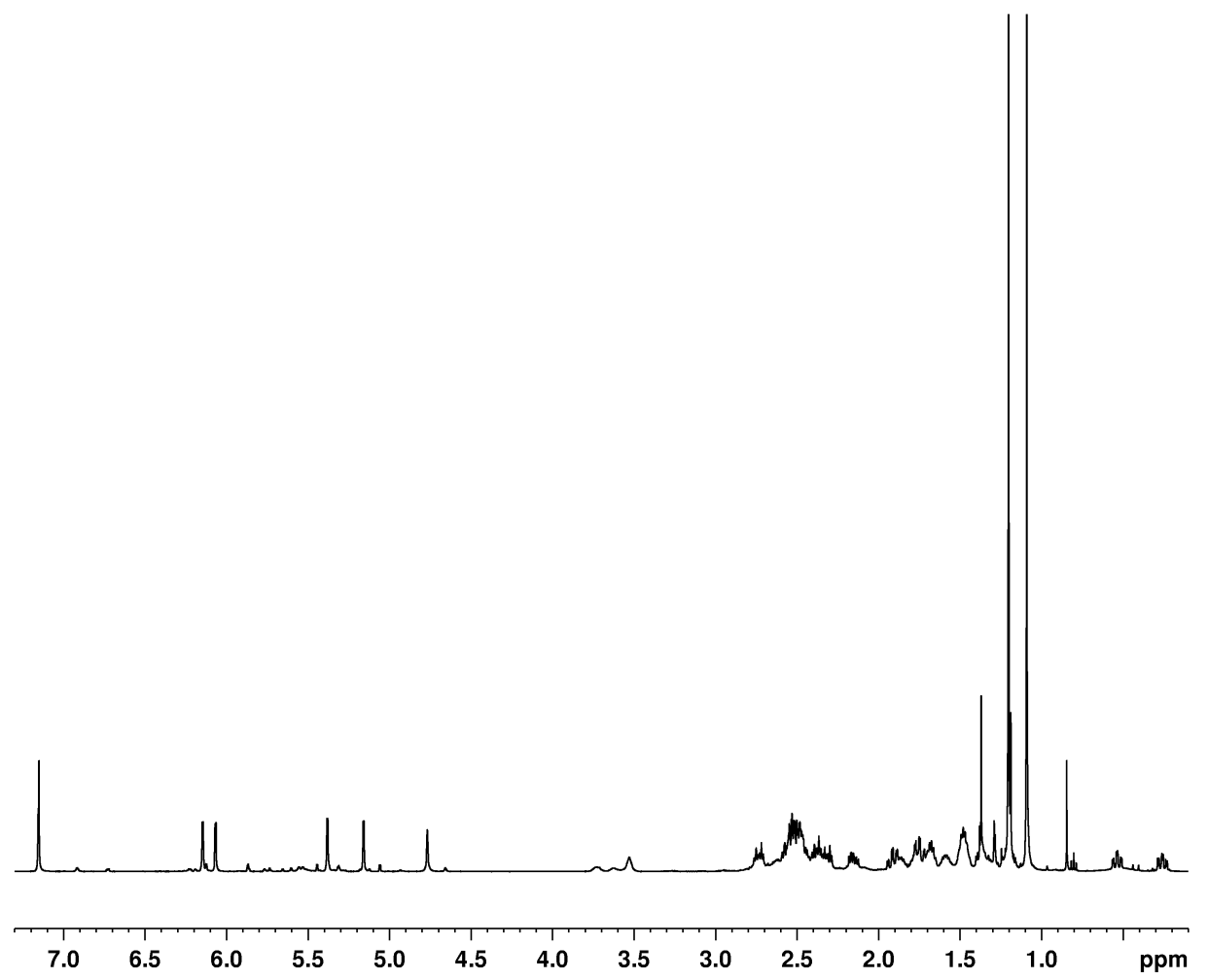

Figure S-8. ${ }^{1}$ H NMR spectrum of isolated complex $\mathbf{1 j}$. 


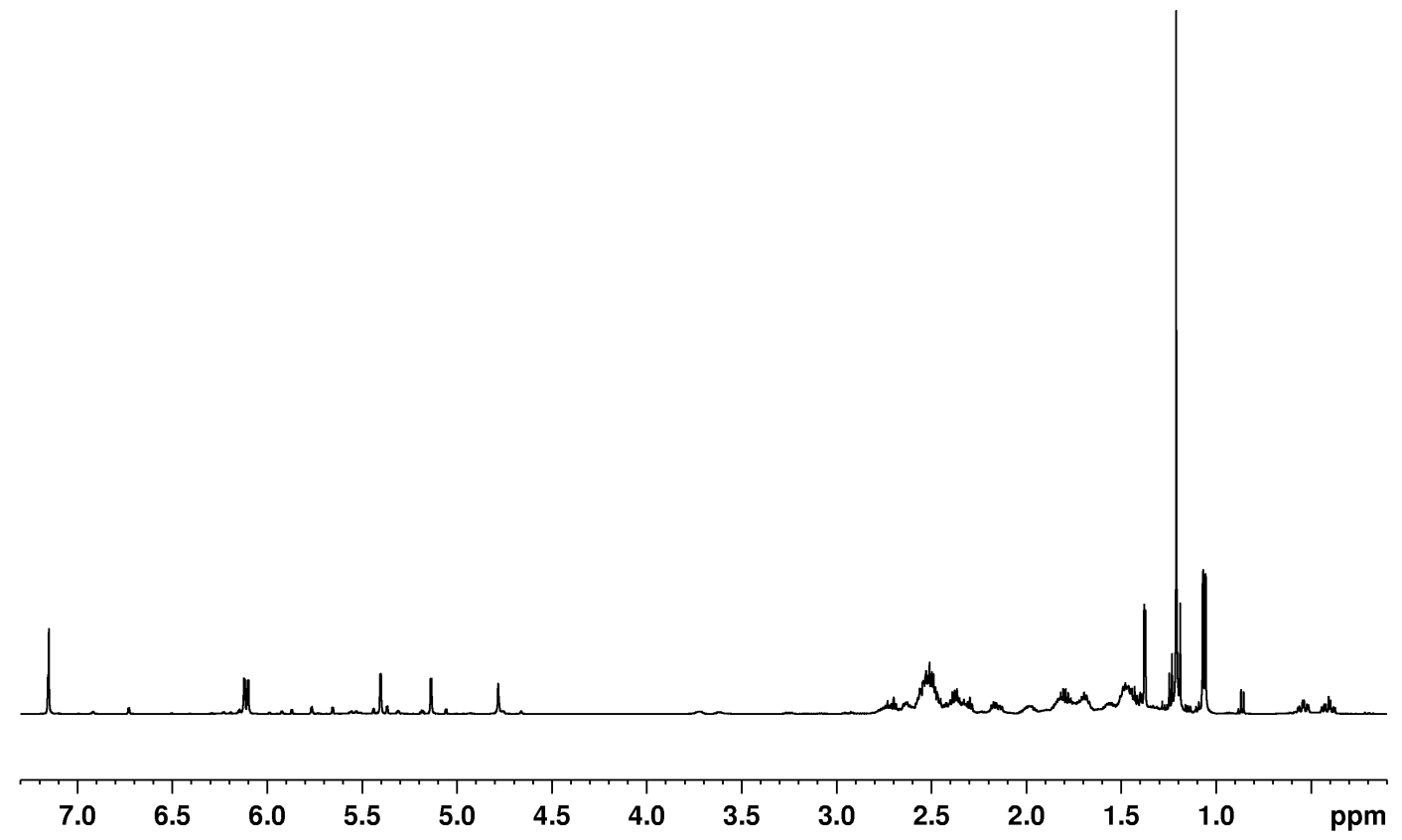

Figure S-9. ${ }^{1} \mathrm{H}$ NMR spectrum of isolated complex $\mathbf{1 k}$.

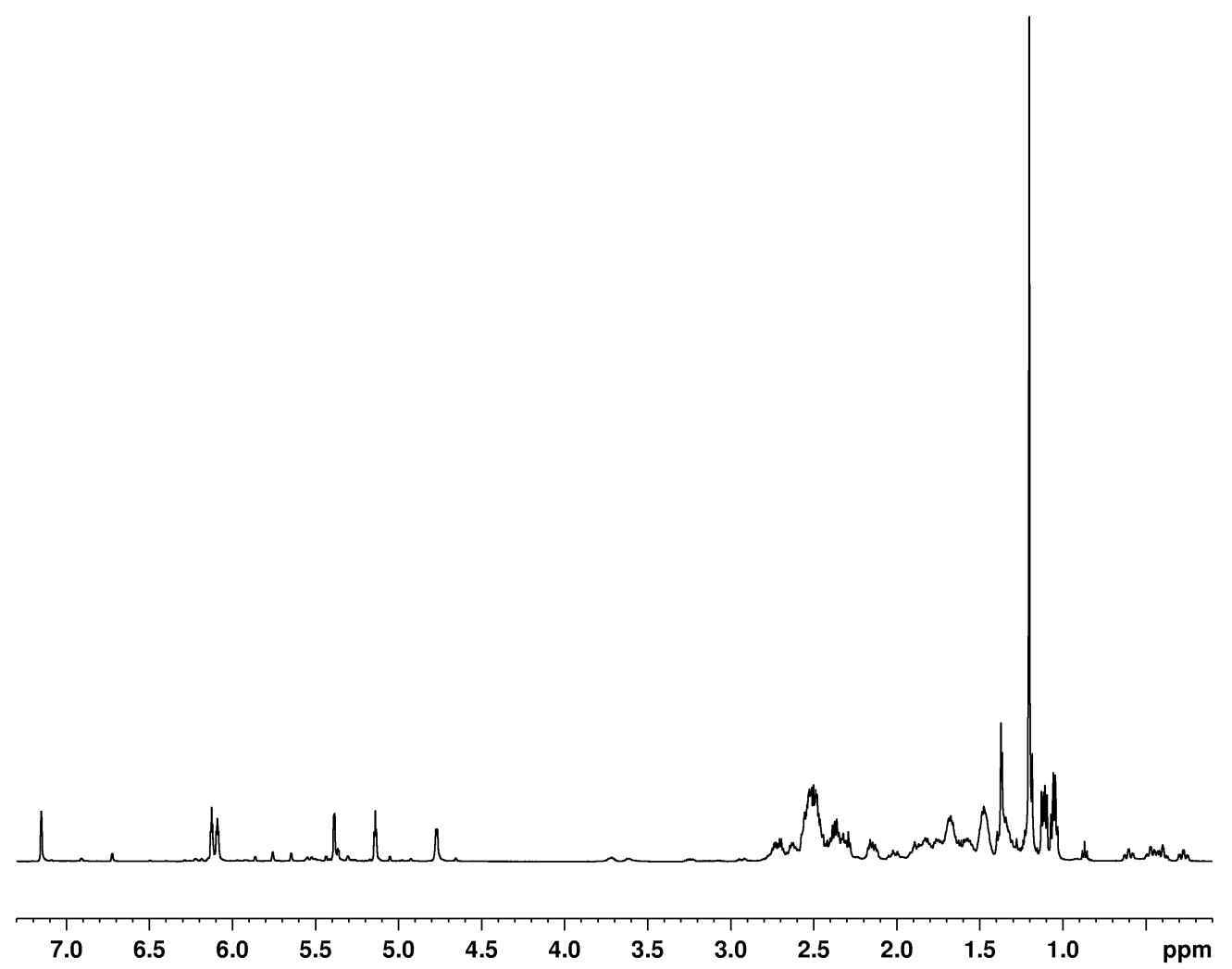

Figure S-10. ${ }^{1}$ H NMR spectrum of isolated complex 11. 


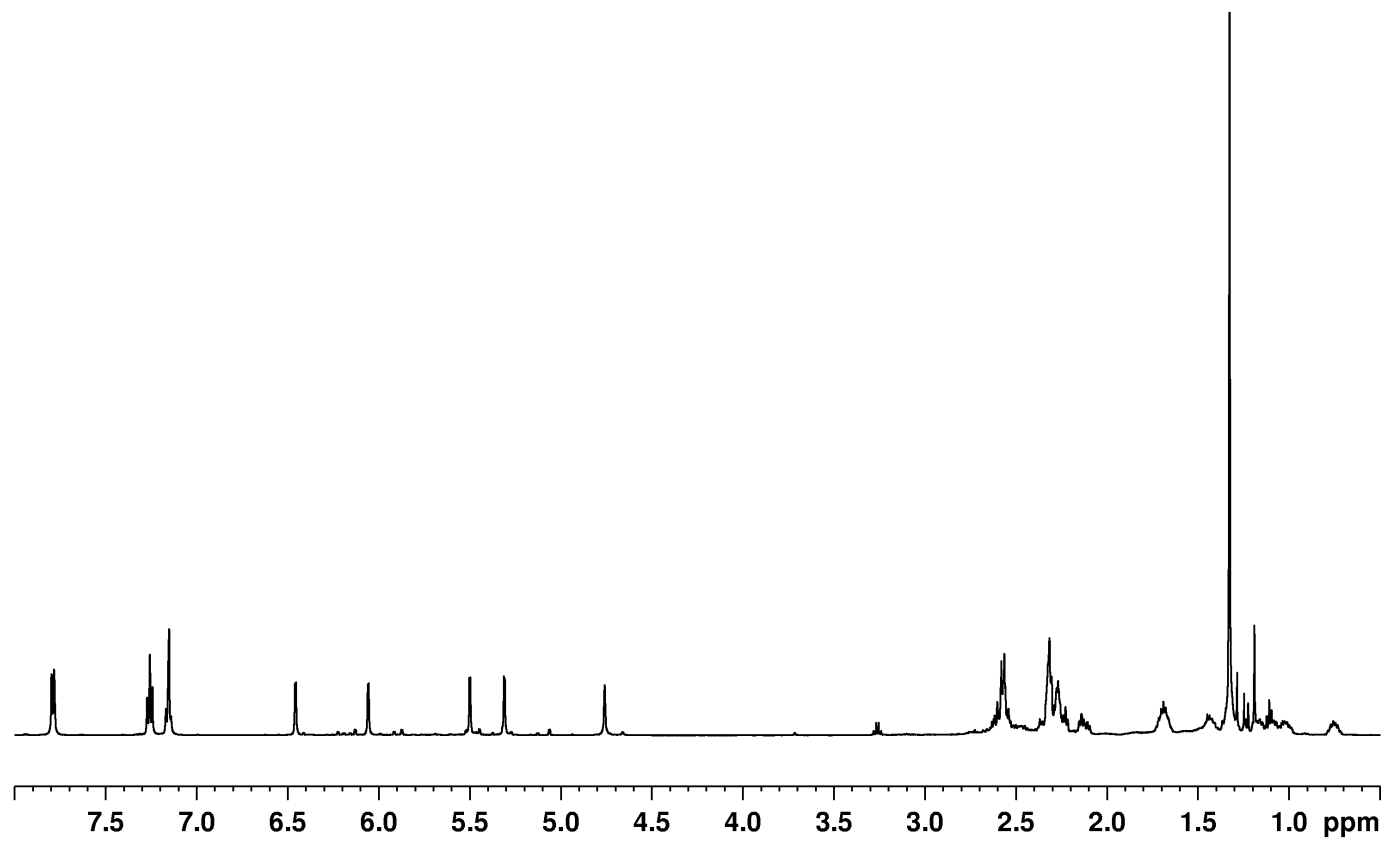

Figure S-11. ${ }^{1} \mathrm{H}$ NMR spectrum of isolated complex $\mathbf{1 m}$.

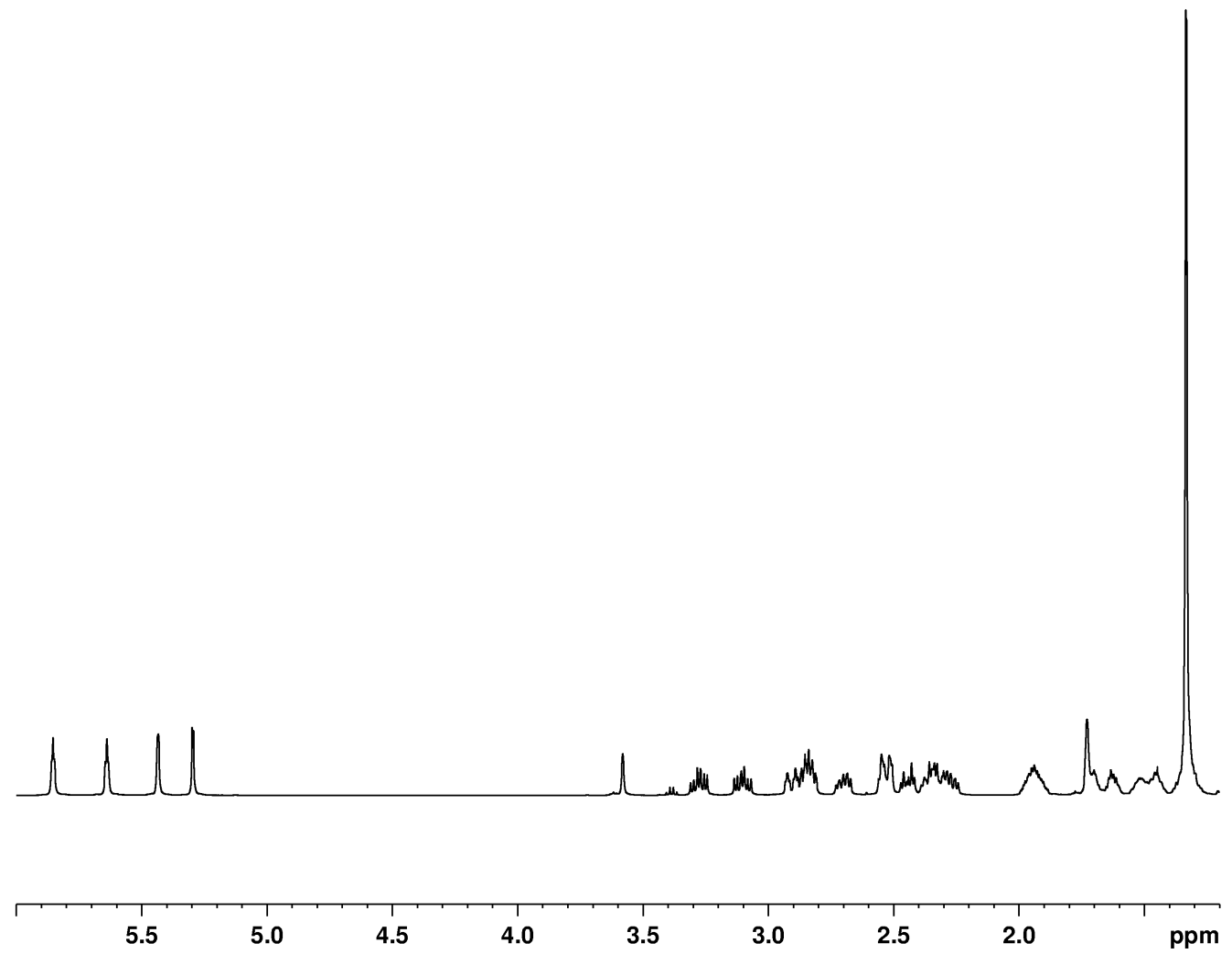

Figure S-12. ${ }^{1}$ H NMR spectrum of isolated complex 5. 


\section{References}

(1) Pangborn, A. B.; Giardello, M. A.; Grubbs, R. H.; Rosen, R. K.; Timmers, F. J. Organometallics 1996, 15, 1518.

(2) SMART: Area-Detector Software Package, Bruker Analytical X-ray Systems, Inc.: Madison, WI, (1995-99).

(3) SAINT: SAX Area-Detector Integration Program, V5.04; Siemens Industrial Automation, Inc.: Madison, WI, (1995).

(4) XPREP:(v 5.03) Part of the SHELXTL Crystal Structure Determination Package, Siemens Industrial Automation, Inc.: Madison, WI, (1995).

(5) SADABS: Siemens Area Detector ABSorption correction program, George Sheldrick, (1996). Advance copy, private communication.

(6) teXsan: Crystal Structure Analysis Package, Molecular Structure Corporation (1985 and 1992).

(7) (a) Sweeney, Z. K.; Salsman, J. L.; Andersen, R. A.; Bergman, R. G. Angew. Chem. Intl. Ed. 2000, 39, 2339. (b) Diamond, G. M.; Jordan, R. F.; Petersen, J. L. J. Am. Chem. Soc. 1996, 118, 8024. 